\title{
SPATIAL DISTRIBUTION MEASUREMENTS OF FISSION NEUTRONS IN WATER AS AN OXYGEN DATA TEST
}

\section{( AWBA DEVELOPMENT PROGRAM)}

FEBRUARY 1978

CONTRACT EY-76-C-11-0014

\section{MASTER}

BETTIS ATOMIC POWLR LABORATORY WEST MIFFLIN, PENNSYLVANIA 


\section{DISCLAIMER}

This report was prepared as an account of work sponsored by an agency of the United States Government. Neither the United States Government nor any agency Thereof, nor any of their employees, makes any warranty, express or implied, or assumes any legal liability or responsibility for the accuracy, completeness, or usefulness of any information, apparatus, product, or process disclosed, or represents that its use would not infringe privately owned rights. Reference herein to any specific commercial product, process, or service by trade name, trademark, manufacturer, or otherwise does not necessarily constitute or imply its endorsement, recommendation, or favoring by the United States Government or any agency thereof. The views and opinions of authors expressed herein do not necessarily state or reflect those of the United States Government or any agency thereof. 


\section{DISCLAIMER}

Portions of this document may be illegible in electronic image products. Images are produced from the best available original document. 
SPATIAL DISTRIBUTION MEASUREMENTS OF FISSION NEUTRONS IN WATER AS AN OXYGEN DATA TEST

(AWBA DEVELOPMENT PROGRAM)

L. Green

J. J. UIIO

CONTRACT NO. EY-76-C-11-0014

FEBRUARY 1978

Printed in the United States of America

Avallable from the

National Technical Information Service

U. S. Department of Commerce

5285 Port Royal Road

Springfield, Virginia 22151

NOTE

This document is an interim memorandum prepared primarily for internal reference and does not represent a final expression of the opinion of Westinghouse. When this memorandum is distributed externally, it is with the express understanding that Westinghouse makes no representation as to completeness, accuracy, or usability of information contained therein.

Operated for the U. S. Department of Energy by WESTINGHOUSE ELECTRIC CORPORATION 
This report was prepared as an account of work sponsored by the United States Government. Neither the United States, nor the United States Department of Energy, nor any of their employees, nor any of their contractors, subcontractors, or their employees, makes any warranty, expressed or implied, or assumes any legal liability or responsibility for the accuracy, completeness, or usefulness of any information, apparatus, product, or process disclosed, or represents that its use would not jnfringe privately owned rights. 


\section{FOREWORD}

The Shippingport A omic Power Station located in Shippingport, Pennsylvania was the first large-scale, central-station nuclear power plant in the United States and the first.plant of such size in the world operated solely to produce electric power. This project was started in 1953 to confirm the practical application of nuclear power for large-scale electric power generation. It has provided much of the technology being used for design and operation of the commercial, central-station nuclear power plants now in use.

Subsequent to development and successful operation of the Pressurized Water Reactor in the AEC'-owned reactor plant at the Shippingport Atomic Power Station, the Atomic Energy Commission in 1965 undertook a research and development program to design and build a Light Water Breeder Reactor core for operation in the Shippingport Station. In $19 \% \mathbf{b}$, with labrication of the Light Water Breeder Reactor (LWBR) nearing completion the Energy Research and Development Administration established the Advanced Water Breeder Applications program (AWBA) to develop and disseminate technical information which would assist U.S. industry in evaluating the LWBR concept. All three of these reactor development projects have been administered by the Division of Naval Reactors with the goal of developing practical improvements in the utilization of nuclear fuel resources for generation of electrical energy using water-cooled nuclear reactors.

The objective of the Light Water Breeder Reactor project has been to develop a technology that would significantly improve the utilization of the nation's nuclear fuel resources employing the well-established water reactor technology. To achieve this objective, work has been directed toward analysis, design, component tests, and fabrication of a water-cooled, thorium oxide fuel cycle breeder reactor to install and operate at the Shippingport Station. Operation of the LWBR core in the Shippingport Station started in the Fall of 1977 and is expected to be completed in about 3 to 4 years. Then the fissionable fuel inventory of the core will be measured. This effort, when completed in about 2 to 3 years after completion of LWBR core operation, is expected to confirm that breeding actually took place.

The Advanced Water Breeder Applications (AWBA) project was initiated to develop and disseminate technical information that will assist U.S. industry in evaluating the LWBR concept for commercial-scale applications. The project will explore some of the problems that would be faced by industry in adapting technology confirmed in the LWBR program. Information to be developed includes concepts for commercial-scale prebreeder cores which will produce uranium-233 for light water breeder cores while producing electric power, improvements for breeder cores based on the technology developed to tabricate and operate the Shippingport LWBR core, and other information and technology to aid in evaluating commercial-scale application of the LWBR concept.

Technical information developed under the Shippingport, LWBR, and AWBA projects has been and will continue to be published in technical memoranda, one of which is this present report. 
Abstract....................................... iv

I. Introduction.................................... I

II. Preliminary Calculations............................ 3

III. Experimental Arrangement.........................

(a) Detectors.................................... 7

(b) Water Tank................................... 9

(c) Neutron Source................................. 9

(d) Detector and Source Support Systems................. 10

IV. Results...................................... II

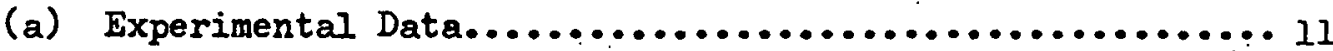

(b) Corrections.................................. 12

( i) Background............................. 12

( ii) $v$-Ray Sensitivity...................... 12

(iii) Count Rate Losses......................... 13

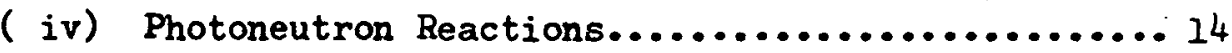

(c) Comparison With Calculations......................... 14

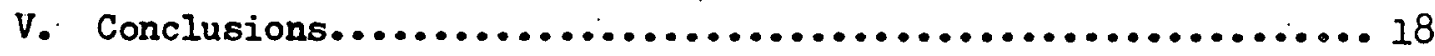

Acknowledgements................................... 20

References....................................... 21

List of Tables..................................... 22

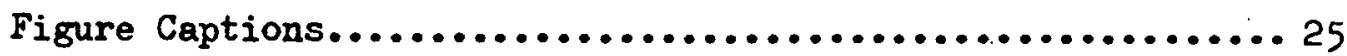

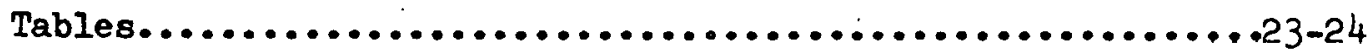

Figures....................................... 27-43 


\section{ABSTRACT}

The spatial distribution of the total neutron density from a ${ }^{252} \mathrm{Cf}$ source in pure water was measured to high statistical precision at distances from 1 to $80 \mathrm{~cm}$ from the source. Assuming the adequacy of the ENDF/B-IV hydrogen, and reasonable constraints on the fission spectrum mean energy, good agreement between experiment and a one-dimensional transport calculation was obtained for both ENDF/B-III and IV oxygen, with Version III slightly better. However, small residual differences remained that could not be removed by adjustment of the spectrum mean energy alone. 


\section{Introduction}

In a program to determine a ouitable cross section set for use in criticality calculations for highly enriched 233 oystems, McNeany and Jenkins ${ }^{I}$ at the Oak Ridge National Laboratory recently reported comparisons of experiment and calculation for a set of six simplo homogeneous assemblies. The assemblies were characterized by Hydrogen/ $233_{0}$ (H/J) ratios varying from zero, for a sphere of bare unreflected ${ }^{23} \mathrm{U}$ metal, to nearly 2000. Two cross section sets were studied, BNDF/B-IV and the 16 group Hansen Roach Library ${ }^{2}$ developed at Los Alamos in 1961. Except for the bare metal assembly, calculations with ENDF/B data showed an upward trend in eigenvalue with decreasing $\mathrm{H} / \mathrm{O}$. The discrepancy reached over $2 \%$ at an $\mathrm{H} / \mathrm{O}$ value of 154. One critical, with an $\mathrm{H} / \mathrm{O}$ of 39, was actually $7 \%$ high. Bowerer, the paraffin reflector of this assembly makes it difficult to interpret. Calculations with the Hansen Roach data set, on the other hand, showed good agreement with exporiment throughout the range of $\mathrm{H} / \mathrm{U}$ ratios. It was concluded that the $\mathrm{BWDF} / \mathrm{B}-\mathrm{IV}$ data file was not satisfactory for the calculation of multiplication factors in highly enriched 233 syotems having $\mathrm{E} / \mathrm{O}$ ratios 1 ess than about 400 .

MeNeany and Jenkins also analyzed two highly enriched $235_{\text {U absem- }}$ blies with the ENDF/B and Hansen Roach data sets. Agreement among the calculations and experiments was good. However, one of the assemblies was of bare metal, a core type which, for ${ }^{233} \mathrm{U}$, gave an anonalously Low eigenvalue result with ENDF/B. The second assombly had an $H / U$ ratio of 1393, for wich good agreement would be expected also for a $233_{0}$ fueled system. Therefore, no real conclusions could be dram from this 
work regarding a possible eigenvalue discrepancy trend for $235_{\mathrm{U}}$ similar to that observed in 2330 systems.

UIlo and Hardy ${ }^{3}$ extended the eigenvalue study of the ENDF/B-IV evaluation to 17 highly enriched homogeneous $233 \mathrm{~J}$ and ${ }^{235} \mathrm{U}$ critical assemblies, including most of those calculated by McNeany and Jenkins. Agreement with the ORNI results was good, with the additional observation of the same upward trend in eigenvalue with decreasing $\mathrm{H} / \mathrm{O}$ for the ${ }^{235} \mathrm{U}$ systems. It was also demonstrated that the eigenvalue error was strongly correlated with the leakage fraction for both fuel types.

A varioty of modifications to the input nuclear data (BNDF/B-IV) were considered by 0110 and Hardy, including fuel, oxygen and hydrogen cross sections, and fission neutron spectra average energy and shape. Eigenvalue sensitivities to changes in each of the data types were evaluated to identify possible candidates contributing to the calculated eigenvalue error. This study revealed that, for high leakage assemblies, the calculated eigenvalues were very sensitive to the oxygen cross sections, as reflected by results for the various ENDF/B versions of oxygen (I, III, and $I V$, since $I$ and II are identical). In particular, the use of $\mathbb{E N D F / B - I}$ oxygen reduced the calculated eigenvalue by approximately $0.6 \%$ compared to ENDF/B-IV, about $1 / 3$ the maximum observed error. Version III showed an intermediate behavior.

The low leakage assemblies were insensitive to the oxygen data set. Thus, in general the calculated eigenvalue dependence on the oxygen data set was shown to be correlated with leakage. This means that, at least to first order, oxygen affects the eigenvalue by modifying core leakage in the direction required to reduce the eigenvalue error. This, coupled 
with the fact that oxygen was a common element in both fuel systems studied, prompted a closer look at the oxygen cross sections.

The present effort was undertaken to provide a clean independent integral test of the ability of the various ENDF/B oxygen evaluations to describe correctly neutron transport in an oxygen containing medium. The work consists of three parts. In the first, a brief calculational survey of neutron transport in various oxygen containing media to identify an optimum one in which to perform the experiment is described. Available integral data for this medium is then discussed. In the second, the present experimental arrangement and its calculational model are then described. Finally, a comparison of experiment and calculation is presented along with the experimental data which can be used for testing any other evaluation.

II. Preliminary Calculations

Any experiment to be considered should be simple from the point of view of analysis, i.e., the geometry should be easily described, and the sensitivity to oxygen data should be reasonable and should not be confounded by uncertainties in the data for other constituents. It was decided that an experiment to measure the neutron distribution in a near infinite oxygen bearing medium from a near point fission source satisfied these conditions. The media chosen for the survey calculations were water, solid $\mathrm{CO}_{2}$, and liquid oxygen.

The calculations were performed for a point source-point detector with PTMG, an extension of $\mathrm{P}_{\mathrm{M}} \mathrm{MG}^{4}$, a one-diriensional $\mathrm{P}_{\ell}$ program. The calculations contained 57 fast groups (the MUFT 54 group scheme with three additional $\Delta u=0.25$ groups above $10 \mathrm{MeV})$. One thermal group $(0.0-0.625 \mathrm{eV})$ with cross sections averaged in a 25 group infinite medium calculation was 
also used. The flux angular treatment was double $P_{1}$ thermally and $P_{7}$ fast. The region of solution extended over $100 \mathrm{~cm}$, and the boundary conditions employed were current $=0$ at the origin and flux $=0$ at the right hand boundary. At the origin was a point fission source with a spectrum that was reported in Ref. 5 .

The results of the calculations are shown in Figs. 1 through 3, where we have plotted the ratio of the thermal neutron flux using ENDF/B-I and III oxygen and an early modified Troubetzkoy ${ }^{7}$ version to that for ENDF/B-IV. The hydrogen and carbon data were taken from ENDF/B-IV for all calculations. Based on these results, water was chosen as the medium to perform the neutron spatial distribution measurements. The reasons for this choice were as follows:

1) Meaningful differences between different oxygen data sets in liquid oxygen do not appear until large distances $(\sim 100 \mathrm{~cm})$ frow the source. Therefore, a large system would have to be constructed, with the attendant problems of handling liquid oxygen.

2) The $\mathrm{CO}_{2}$ systen would also have to be large to detect a difference between oxygen data sets. In addition, the analysis would be complicated by the presence of the carbon scattering resonances, where characterization and uncertainties might confound the oxygen data comparisons.

3) The hydrogen cross section is well known. Water is a convenient medium from an experimental viewpoint, with the important parameters of purity and density (i.e., temperature) easily controlled. In addition, preliminary tests with an available ${ }^{252} \mathrm{Cf}$ spontaneous fission source indicated that the required statistical accuracy of $\pm 1 \%$ beyond $50 \mathrm{~cm}$ could be achieved with a thermal detector. 
Calculations similar to those above for each of the three media were carried out at indium and gold resonance energies. An enhanced sensitivity to oxygen was observed. However, this advantage was outweighed by the much lower flux levels.

The literature is replete with reports of neutron distribution measurements in pure water, and it is of interest to examine these for application to the problem at hand, i.e., determination of a best available data set for oxygen. The measurements reported are generally of two types, age and spectrum shape.

The age is proportional to the second apatial monent of the flux distribution (generally determined at indium resonance energy, $1.46 \mathrm{eV}$ ) from a point or plane source (with point or plane detector). The contribution to the second moment falls off very rapidly with distance beyond a few centimeters from the source, with over $90 \%$ of the moment integral accumlated within $20 \mathrm{~cm}$. The emphasis in age measurements is therefore on relatively short distances. In this region, the flux distribution is not very sensitive to scattering from oxygen and, in fact, the fission age in water decreases only about $6 \%$ if oxygen slowing down is left out completely. Tabie I lists the ages calculated using PMG for a ${ }^{252} \mathrm{Cf}$ fission source ${ }^{5}$. for three ENDF/B oxygen evaluations. Only the differences are significant here, and these are seen not to exceed about 1\%. This is less than the experimental uncertainty for the best known flux age, that from a ${ }^{235}$ fission source. Therefore, neutron age results cannot be used to provide a conclusive test of the adequacy of different oxygen cross section sets. While age results alone are not useful here, Spiegel ${ }^{6}$, in a recent measurement of the age of ${ }^{252} \mathrm{Cf}$ fisgion neutrons in water, took indiun 
activation data out to $65 \mathrm{~cm}$. His paper lists the results of a smooth curve fit to the normalized data at $0.5 \mathrm{~cm}$ intervals. An attempt was made to analyze these data to determine if some information about oxygen could be extracted. Calculations were performed again with P7MG. The source encapsulation was described as an aluminum sphere of radius $0.435 \mathrm{~cm}$, with the ${ }^{252} \mathrm{Cf}$ fission neutrons originating from a region at the center with a $1 \mathrm{~mm}$ radius. The $P_{0}$ flux in multigroup $54(1.855-1.439$ eV) was computed at 0.5 cm intervals along a radius from the source and compared with experiment. The fission spectrum in Ref. 5 was employed. Four sets of calculations were performed using different oxygen data sets: ENDF/B-I, III, IV and the previously mentioned evaluation by Troubetzkoy? . Enploying the same nomalized units for experiment and calculation, the ratios of experiment to calculation are shown plotted in Fig. 4. It can be seen from the figure that good agreement for the spatial distribution of indium resonance neutrons is not achieved for any of the oxygen data sets.

The sensitivity of these calculations to the choice of a ${ }^{252} \mathrm{Cf}$ fission neutron spectrum representation was next investigated. The calculation was repeated using the $\mathrm{NNDF} / \mathrm{B}-I$ oxjgen and a Maxwellian representation with the same average energy as that of the complex spectrum of Ref. 5, 2.10 MeV. As shown in Fig. 5, no significant difference was found. The average energy of the Maxwellian was then raised to 2.154 MeV, which was judged to be a harder spectrum than the upper limit of the error. The results are displayed in Fig. 5; signifiant differences still remain. It was concluded that no reasonable hardening or change in shape of the ${ }^{252}$ Cf fission neutron spectrus would produce good experiment/calculation agreement over the epatial 
range epanned by Spiegel's magurements. The reasons for this are not known.

Spectrun measurements in good geometry have proven useful in the testing of total cross section data $9,10,11$. However, in evaluating the ability of an oxpgen data set to describe leakage correctly, scattering angular distributions are important, and any experiment to be considered should be sensitive to them. Some of the spectrum measurements also required unfolding of a proton recoil distribution and resolution broadening of the calculation before a comparison with calculation could be made. These procedures introduce significant uncertainties.

Based on the above considerations, it was decided that a new experiment that would emphasize the deep penetration of neutrons in water to an accuracy of $\sim \pm 1 \%$ at distances out to $70-75 \mathrm{~cm}$ was needed. In this way (assuming the adequacy of ENDF/B-IV hydrogen and reasonable constraints on the shape and mean energs of the ${ }^{252}$ (f spectrum), experiment/calculation comparisons could be used to assess the adequacy of different oxygen cross section data sets.

III. Experimentel Arrangement

(a) Detectors

Since the previously described calculations indicated significant Bensitivity to oxygen scattering of the thermal neutron flux distribution in water, it was decided to use a therwally sensitive detector. The advantages of this choice compared to a resonance detector are high efficiency and the option of using a "live" system, i.e., a neutrom counter rather than the activation of a foil. This, again, permits a higher rate of data accumiation. The requirements for the detector 
are that it be small to minimize water displacement, have adequate efficiency, and very low y-ray sensitivity. The associated electronics should also have a high count rate capability (which is, of course, closely related to pray sensitivity). In addition, it should be possible to represent accurately the enersy dependence of the detector efficiency in the calculation.

All of these requirements were met using miniature boron lined neutron detectors with a fast electronics system. The specifications for the detectors were determined from preliminary tests with available detectors and ${ }^{252} \mathrm{Cf}$ sources. Three detectors were fabricated, designated as the high efficiency, low efficiency, and zero efficiency detectore. Bxcept for the borom lininge, all wore ade of idontical enterials and had the ans disonations, $0.635 \mathrm{on}$ dienoter with 0.635 $\mathrm{cm}$ active length. The high sensitivity detector, which covered the distance range of 22 to $80 \mathrm{~cm}$ from the source, had an enriched ( $92 \%$ ) ${ }^{10} \mathrm{~B}$ lining $0.2 \mathrm{mg} / \mathrm{cm}^{2}$ in thickness. The low sensitivity detector, with a depleted ( $11 \%$ ). ${ }^{10} \mathrm{~B}$ lining approximately $0.1 \mathrm{mg} / \mathrm{cm}^{2}$ thick, covered the distance range of 11 to $45 \mathrm{~cm}$. The detector sensitivities, as provided by the manufacturer, were $7.2 \times 10^{-3}$ and $3.3 \times 10^{-4} \mathrm{c} / \mathrm{s}$ per unit incident moderated neutron flux. Both linings were optically thin to thermal neutrons so that the energy dependence of the deteotor efficiencies corresponds to the shape of the ${ }^{10} \mathrm{~B}$ absorption cross section. This allowed a simple representation of the detector in the calculation, as discussed below.

The zero sensitivity detector had no boron lining and was used to determine the $\gamma$-ray response. This is of special importance 
since even a low Yray sensitivity, caused primarily by pulse pileup, could seriously distort the measured neutron distribution.

It should be pointed out that the ${ }^{10} 0_{B}$ cross section is accurateIy $1 / V$ in shape throughout the energy range of importance here. Therefore, this experiment weasured the spatial distribution of the total neutron density, independent of the slowing down spectrum shape at each measurement location.

The detectors were placed at the bottom of thin walled $(0.50 \mathrm{~mm})$ zircaloy tubes $0.77 \mathrm{~cm}$ in diameter and $152 \mathrm{~cm}$ long. Low capacitance cable coupled the detectors to a fast rise time (16. ns), short clipping time ( $150 \mathrm{ng}$ ), charge sensitive preamplifier designed specifically to reduce $\gamma$-ray pulse pileup in high $\gamma$-fields. This preamlifier had the additional advantage of very low input impedance, thereby accomodating long cable lengths at the input. The output of the preamplifier went directly to a fast, fixed threshold discriminator.

\section{(b) Water Tank}

The experiment was performed in a cylindrical stainless steel water tank, $229 \mathrm{~cm}$ in diameter and $183 \mathrm{~cm}$ high. The tank was filled with deionized water, wich was continuously circulated through a multibed resin colum to maintain mater purity. Water temperature, as measured by an alcohol thermometer was $20.5 \pm 0.2^{\circ} \mathrm{C}$ and was controlled to within $0.1^{\circ} \mathrm{C}$ by means of a proportional temperature controller which operated a 1500 watt heater.

(c) Neutron Source

The ${ }^{252} \mathrm{CP}$ spontaneous fission isotope was contained in a standard Oak Ridge National Laboratory NSB encapoulation, as shown in Fig. 6. 
Also included in the figure is all of the information on encapsulation dimensions and materials as provided by $0 \mathrm{RNL}$. The ${ }^{252} \mathrm{Cf}$ content at the time of those measurements was $1.3 \mathrm{mg}$. The largest contribution to the dimensional uncertainty in this experiment was the radial location of the ${ }^{252} \mathrm{Cf}$ within the resin. For purposes of experiment/ celculation comparieons, it was asmumed that the $252 \mathrm{Cf}$ was located at the center with a radius of $1 \mathrm{~mm}$. The source was threaded into a $1.90 \mathrm{~cm}$ high stainless steel plug pressed into the end of a thin walled zircaloy tube $2.0 \mathrm{~cm}$ in diameter and $200 \mathrm{~cm}$ long. Holes drilled into the tube allowed it to fill with water.

(d) Detector and Source Support Systems

The source and detector tubes were rigidly suspended from platforms placed on a pair of heavy aluminum rails $12.7 \mathrm{~cm}$ high and $3.8 \mathrm{~cm}$ wide resting on the $9 \mathrm{~cm}$ wide flange on the top of the tank. Machining precision was kept within $\pm 0.025 \mathrm{~mm}$ for all important surfaces to insure good parallelism and perpendicularity. These neasures were necessary since the distance scale was set at only a single location.

The detector platform rode on 4 nylon whesls whose larger central diameter fit snugly into the rail Rrooves while the smaller diameter inner and outer edges rested on the rails. Holes 3 in diameter were precision drilled every centimeter along the top of both rails. Close fitting steel pins passing through the platform and into the holes provided positive locking for good positional reproducibility.

The distance scale was set by placing the source platform so that the source was about $76 \mathrm{~cm}$ from the tank wall (1/3 of the tank diameter) and $90 \mathrm{~cm}$ below the water surface. The detector platform 
was then wheled in close to the source and the detector height varied until the maximum count rate location was found. This was done separately for each detector, and insured that the source and detector were at the same effective heights. An aluminum rod with a hole drilled through its center, such that the detector tube fit through snugly, was then placed at the detector location. The rod length had been accurately machined so that the distance from the center of the source encapsulation to the detector center line, when the rod end made electrical contact with the ourface of the source, was $17 \mathrm{~cm}$. Contact was made by rarying the source platform location with the detector platform pinned in place. The source platform was then clamped to the rails. The estimated uncertainty in this procedure was $\pm 0.5 \mathrm{~mm}$.

\section{Results}

\section{(a) Experimental Data}

Data were taken every centimeter, from 11 to $45 \mathrm{~cm}$ for the low efficiency detector and 22 to $80 \mathrm{~cm}$ for the high efficiency detector. Statistical precision was limited by drift in the electronics which required frequent normalizations at standard locations. Runs were repeated until the standard deviation at each point was $1 \%$ or better. The count rate data in the overlap region from 22 to $45 \mathrm{~cm}$ were ratioed point by point, and a mean and standard deviation for the high to low efficiency detector of $21.900 \pm 0.215$ were obtained. The standard deviation 1s cohsistent with the single measurement precision for the ratio and it was concluded that, within statistics, no shape difference was observed between the two detectors in the overlap region. 
The moan is also in excellent agreement with the value of 21.8 obtained from the ratio of the detector sensitivities provided by the manufacturer.

The results for the combined set of data, normalized to the high efficiency detector, are listed in Table II and plotted in. Fig. 7. No smoothing has been performed. The data are fully corrected, as indicated below, and may be directly compared with calculation.

(b) Corrections.

(. i) Background

By background, we mean counts due to electronic noise, cosmic rays, etc. A linear least squares fit to the In (Ar ${ }^{2}$ ) data of Table II from 71 to $80 \mathrm{~cm}$ was made to obtain a relaxation length in this region. This was found to be $8.84 \mathrm{~cm}$. Data were also accumulated at $104 \mathrm{~cm}$, the farthest location the detector platform could be accurately located. This location is still $50 \mathrm{~cm}$ from the tank wall and a factor of over 20 lower in eignal thon the $80 \mathrm{~cm}$ point. An excess of $0.0006 \mathrm{c} / \mathrm{s}$ over that predicted for $104 \mathrm{~cm}$ from the relaxation length was observed and attributed to "background". No attempt was made to assign an uncertainty to this value since, as can be seen from Table II, the maximum correction is only about $0.5 \%$.

(ii) Y-Ray Sensitivity

The pray response was found to be a strong function of detector bias, as illustrated in Fig. 8. The operating biases of the two neutron eensitive detectors, shown in Fig. 8, 
were a compromise between low $y$-ray response and the need to operate on the detector plateaus to minimize drift. The spatial dependence of the v-ray response at 875 volts bias, corresponding to the low sensitivity detector, is show in Fig. 9. The ratios of the zero sensitivity to low sensitivity detector responses at each of the measurement points are also shown in Figure 9. The statistical uncertainty for the three points at 30,35 and $40 \mathrm{~cm}$ is about 20\%. Within measurement precision, it can be seon that the ratios are constant at approximately $0.6 \%$. This is a surprising result and is evidently caused by a balance between rapidly decreasing neutron and pelse pileup sigeals. This ratio would be a factor of over 50 lower for the high sensitivity detector. Therefore, no $Y$-ray response correction applied to either of the neutron sensitive detectors.

\section{(iii) Count Rate Losses}

The maximam count rate encountered in this experisent was $2768 \mathrm{o} / \mathrm{s}$ for the high sensitivity detector at $22 \mathrm{~cm}$. The expected count rate 108 s at this value, based upon measurements taken with a critical assembly on a stable period, was $0.05 \%$, with an estimated uncertainty of 25\%. This is negligible compared to the statistical uncertainty, so no corrections were made. A check on this procedure is provided by the excellent shape agroement in the overlap region of the high and low Bensitivity detectors. 


\section{(iv) Photoneutron Reactions}

At long distances from a fission source in water, photoneutrons from $(v, n)$ reactions on the deuteriun in the water become significant ${ }^{12}$. However, at $80 \mathrm{~cm}$, the contribution is estimated to be only about $0.1 \%$, so no correction for this effect was necessary.

\section{(c) Comparison With Calculation}

Tu culvar with the experimental data in Table II, 57 group P7MG calculations were performed similar to the survey calculations described earlier. The major differences were in the source and detector descriptions. The source dinensions and compositions as represented in the calculation are shown in Fig. 10. The $1.38 \mathrm{~cm}$ outer radius was obtained by sphericizing the source body and plug into which it was threaded. Similarly, the pressed aluminum pellet was sphericized to form the intermediate radius. The resin was distributed homogeneously in the pressed aluminum, and figsion neutrons originated within the $0.1 \mathrm{~cm}$ radius about the center.

The calculations included a trace $\left(10^{-15}\right.$ atoms/b-cm) of $10_{B}$ uniformly distributed throughout the water. The ${ }^{10}{ }_{B}$ absorption fraction was then calculated every $\mathrm{cm}$ as a function of distance from the source. In the region of solution, no mesh spacing was greater than $0.385 \mathrm{~cm}$. This spacing was found to be adequate based on a mesh sensitivity study. The quantity calculated is given by

$$
A^{B 10}=\int_{E} \int_{V} \Sigma_{a}^{B 1 Q}(\Sigma) \phi(r, B) d E d \bar{r}
$$

Due to the $1 / N$ absorption cross section of the ${ }^{10} \mathrm{~B}, \mathrm{Eq} \cdot$. (1) can be 
rewritten as:

$$
\begin{aligned}
A^{B 10} & =N^{B 10} \sigma_{a_{0}}^{B 10} \int_{E V} \int_{V} n(E, r) d E d \bar{r} \\
& =4 \pi N^{B 10} \sigma_{\sigma_{0}}^{B 10} \int_{E} \int_{R_{i}}^{B i+1} n(E, r) d E r^{2} d r .
\end{aligned}
$$

The energy integration is over the range from 0.0 to $21.0 \mathrm{MeV}$ and the volume integration in each case is over the spherical shell between $R_{i}$ and $R_{i}+1$, where $\left(R_{i}+1-R_{k}\right)=0.77 \mathrm{~cm}$. This is just the diameter of the detector tube. Dividing Eq. (2) by the volume yields. the volume normalized $10_{B}$ absorption rate

$$
A^{B 10}\left(\frac{R_{i}+R_{i+1}}{2}\right)=N^{B 10} \sigma_{a_{0}}^{B 10} \text { भ }\left(\frac{R_{i}+R_{1}+1}{2}\right)
$$

where

$$
\eta\left(\frac{R_{i}+R_{i}+1}{2}\right)=\frac{3 \cdot \int_{E} \int_{R_{i}}^{R_{i}+1} n(E, r) r^{2} d r d E}{\left(R_{i+1}^{3}-R_{i}^{3}\right)}
$$

Is the spatially averaged total neutron density wich is assigned to the midpoint of the spherical shell. Hence, the calculations yield a quantity which is directly proportional to the total neutron density as a function of radius and which is directly comparable to the measurements.

Before presenting comparisons with the various oxygen data sets, Fig. 11 11lustrates the strong dependence of neutron density on oxygen scattering at increasing source-detector distances. For this calculation, oxygen scattering was deleted completely, and the 
EXP/CAIC ratio was normalized at $13 \mathrm{~cm}$. As expected, the calculation increasingly over-predicts the density, with the error reaching over a factor of 6 at $80 \mathrm{~cm}$.

In Fig. 12, we show the EXP/CAIC comparison for the early modified Troubetzkoy ${ }^{7}$ evaluation. The data have been normalized in this, as well as all subsequent, comparison in the $13-20 \mathrm{~cm}$ region. The first two points were not included in the normalizations since the slowing down curve (Fig. 7) shows increased curvature here. This indicates that those points would be more sensitive to the source location and its details. It can be seen in Fig. 22 that the EXP/CALC ratio is poor beyond $40 \mathrm{~cm}$ with the discrepancy increasing to $20 \%$ near $70 \mathrm{~cm}$. The inadequacy of this oxygen evaluation has been observed by others 10,13

The striking improvement obtained for ENDF/B-I oxygen is shown in Fig. 13. The cross sections of importance here were taken by WNDF from the work of Slaggle and Reynolds ${ }^{14}$. These cross sections were maintained in $\mathrm{FNDF} / \mathrm{B}-\mathrm{II}$. Agreement between experiment and calculations is seen to be excellent out to about $50 \mathrm{~cm}$ with the calculation over-predicting slightly the neutron density beyond this distance.

The results for ENDF/B-III and IV are shown in Figs. 14 and 15 respectively. Two observations may be made from these figures. Pirst, except for the blightly faster rise near $25 \mathrm{~cm}$ and above 75 fm for ENDF/B-IV, they are very close in shape. Secondly, both evaluations systematically underpredict the neutron density by $1.5-2 \%$ beyond $30 \mathrm{~cm}$. The relative behavior of all three evaluations is, 
incidentally, consistent with their eigenvalue response, i.e., a higher neutron density further out which is indicative of increased leakage and lower eigenvalue.

Since. we have standardized the use of ENDF/B-IV hydrogen cross sections in this work, the only other variable that can affect the EXP/CALC comparisons is the characterization of the ${ }^{252}$ Cf fission neutron spectrum. The spectrum model used in all calculations above was that reported by Green et $\underline{\text { al }}^{5}$. It has a complex shape with a mean energy of $2.105 \mathrm{MeV}$. This is at the low end of the range of measurements and is significantly softer than the reference Maxwellian average energy of $2.13 \mathrm{MeV}$ proposed in the recent NBS evaluation ${ }^{15}$. It was judged that the most likely range of mean energies for the ${ }^{252} \mathrm{Cf}$ spectrum is $2.10<\overline{\mathrm{E}}<2.13 \mathrm{MeV}$. The effect of increasing the mean energy is to worsen the $\mathrm{GNDF} / \mathrm{B}-\mathrm{I}$ comparison while improving those for Versions III and IV. For this reason we consider Versions III and IV to be in better agreement with the present experiment than Version I oxygen.

The effect of changes in mean energy and shape of the fission neutron spectrum were investigated for Version IV oxygen by calculations using Maxwellian distributions at the estimated limits of the mean enerBy values of 2.10 and $2.13 \mathrm{MeV}$. The lower value also corresponds to the same mean energy as the complex spectrum model and provides a measure of the shape sensitivity. The results are shown in Fig. 16. Comparison of Fig. 16 with Fig. 15 shows the shape change to have a small effect with a maximum difference in the EXP/CAIC ratio of about $1 \%$ near $40 \mathrm{~cm}$. Of far greater importance is the mean energy change. For 
the $27 \mathrm{keV}$ difference the ratio is seen to swing from below to above unity, reaching values of 0.97 and 1.02 , for a difference of 5\% beyond $70 \mathrm{~cm}$. Therefore, for a reasonable choice of limits on the mean energy of the ${ }^{252}$ Cf fission spectrum, Version IV oxygen brackets the experimental results.

In Fig. 17, we show the EXP/CALC ratio for an intermediate energy $(\bar{E}=2.118 \mathrm{MeV})$ Maxwellian. Agreement is seen to be within $\pm 1.5 \%$ over the entire range from 11 to $80 \mathrm{~cm}$. Comparison of Figs. 14 and 15 shows that Version III oxygen will behave similarly, although with a significantly smaller bulge between 25 and $40 \mathrm{~cm}$, so that agreement will be about $\pm 1 \%$. The agreement between experiment and calculation using both ENDF/B III and IV must be considered excellent in view of the change in magnitude of nearly 4 decades in the neutron density. Nonetheless, it is apparent from F18. 17 . that systematic differences between experiment and calculation do exist, and these differences may be a result of the inadequacy of the Maxwellian description for the ${ }^{252} \mathrm{Cf}$ fission neutron spectrum.

\section{Conclusions}

The spatial distribution of the total neutron density from a near point fission ${ }^{252} \mathrm{Cf}$ neutron source in pure water has been measured every $\mathrm{cm}$ from 11 to $80 \mathrm{~cm}$ to a statistical precision of better than $1 \%$. The purpose of the measurements was to provide data for testing the ability of various oxygen evaluations to describe adequately neutron leakage in oxygen containing systems. The measurements were compared to spherical geometry multigroup transport calculations. Assuming the adequacy of the ENDF/B-IV hydrogen and a reasonable constraint on the ${ }^{252} \mathrm{Cf}$ fission 
spectrum mean energy, good agreement between experiment and calculation was obtained for both ENDF/B-III and IV oxygen with Version III slightly better. A shift of $\pm 13 \mathrm{keV}$ in mean energy produced strong disagreement at long penetration distances for both evaluations.

The remaining differences between experiment and calculations for both Versions III and IV oxygen, while small, are systematic and cannot be removed by adjustment of the fission neutron spectrum mean energy alone. This may. be due to inadequacy of the Maxwellian description of the fission neutron energy distribution. 
$-20=$

Acknowledgements

The authors wish to express their gratitude to $\mathrm{Dr}$. N. M. Steen for his advice and encouragement in the work. 


\section{References}

1. S. R. MCNeany and J. D. Jenkins, "Comparison of Hansen Roach and ENDF/B-IV Cross Sections for 2330 Criticality Calculations," ORNL-TM-5113, Oak Ridge National Laboratory (1976).

2. G. E. Hansen and W. H. Roach, "Six and Sixteen Group Cross Sections for Fast and Intermediate Critical Assemblies," LAMS-2543, Los Alamos Scientific Laboratory (1961).

3. J. J. OIlo and J. Hardy, Jr., "Analysis of Homogeneous $233 \mathrm{~V}$ and 235 Critical Assemblies with ENDF/B-IV Data," WAPD-TM-1299, Bettis Atomic Power Laboratory (1977).

4. H. Bohl, Jr. et al., "P3MG-1, A One Dimensional Multigroup P-3 Program for the Philco-2000 Computer," WAPD-TM-272, Bettis Atomic Power Laboratory (1963).

5. L. Green, J. A. Mitchell and N. M. Steen, Nucl. Sci. Ing., 20, 257 (1973).

6. V. Spiegel, Nucl. Sci. Nag., 54, 28 (1974).

7. E. S. Troubetzkoy, "Final Report Covering the Period 17 Dec. 1958 30 Sep't. 1959. Fast Neutron Cross Sections of Iron, Silicon, Aluminum, and Oxygen," NDA-2111-3, Vol. C, Nuclear Development Corporation of America (1959).

8. N. R. Candelore and R. C. Gast, "RECAP-3, A Monte Carlo Program for Estimating Epithermal Capture Rates in Rectangular or $60^{\circ}$ Parallelogram Geometry," WAPD-TM4-437, Bettis Atomic Power Laboratory (1964).

9. V. V. Verbinski, M. S. Bokchari, J. C. Courtney, and G. E. Whitesides, Nuol. Soi. Ang-, 27, 283 (1967).

10. C. E. Clifford et 르, Nucl. Sci. Engo, 27, 299 (1967).

11. L. Harris, Jr., G. Shorwood, and J. S. King, Nucl. Sci. Ing., 26, 571 (1966).

12. B. T. Price, C. C. Horton, and K. T. Spinney, Radiation Shielding, pp. 319-321, Macmillan Co., New York (1957).

13. R. W. Stooksberry and E. E. Carroll, Jr., Nucl, Sci. Rng., 30, 213 (1967).

14. E. L. Slaggie and J. T. Reynolds, "16 0 Fast Neutron Cross Sections and Legendre Moments," KAPL-M-6452, Knolls Atomic Power Laboratory (1965).

15. J. A. Grundl and C. M. Elsenhauer, "Fission Spectrum Neutrons for Cross Section Validation and Neutron Flux. Transfer," Nuclear Cross Sections and Technology Conference, MBS Special Publication 425, p. 250 (1975). 
$-22-$

List of Tables

Table No.

I

II
Title

Calculated Ages for Different ENDF/B Oxygen Evaluations

Count Rate Data
Page No.

23

24 
Table I

CAICULATED AGES FOR DIFFERENT ENDF/B OXYGEN EVALUATIONS ${ }^{2}$

Version

I

II

III
Ages $\left(\mathrm{cm}^{2}\right)$

27.1

26.9

26.8

a Fission spectrum from-Ref. 5. 


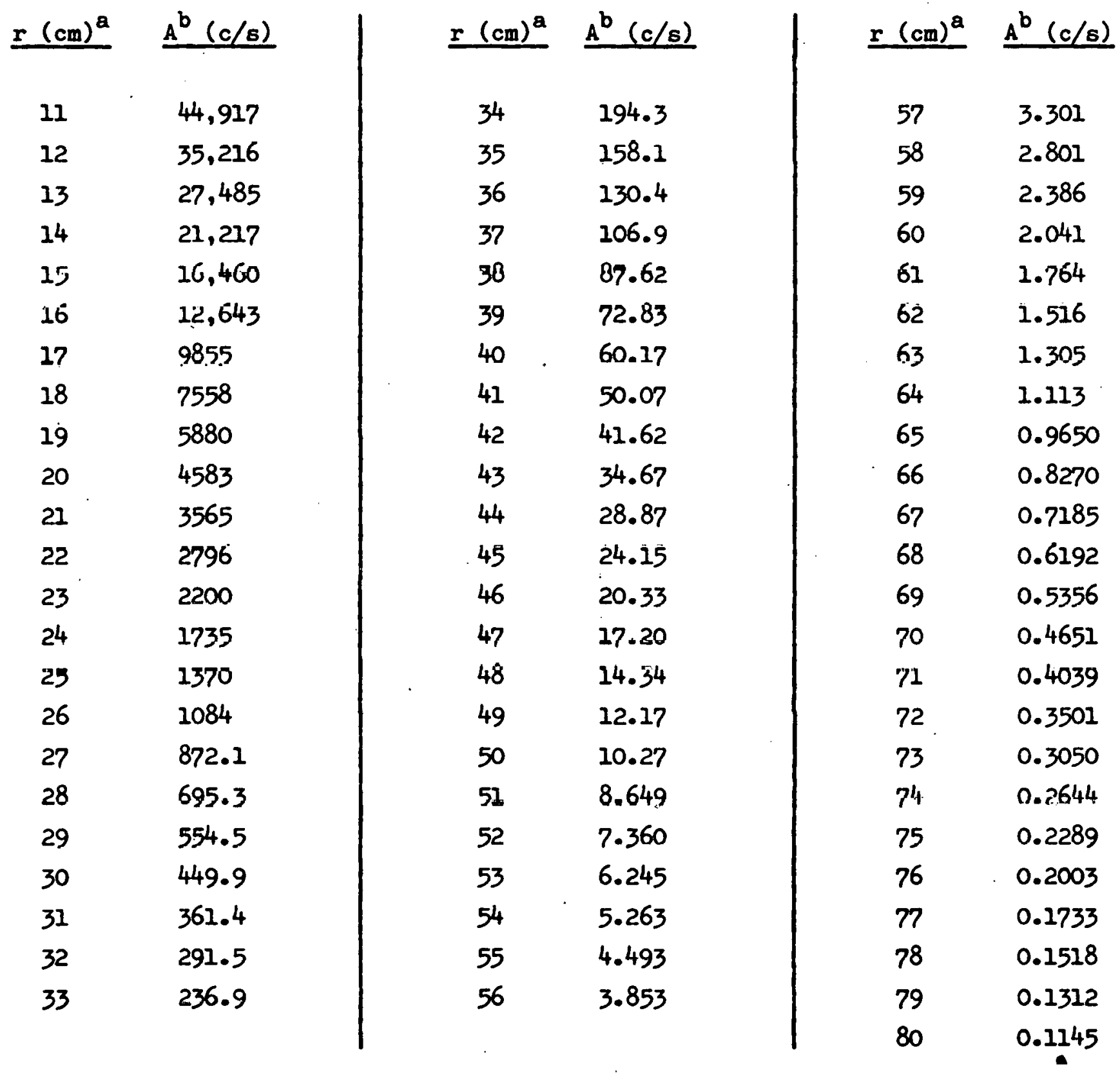

a Distance between centerline of detector and source.

b Count rate normalized to the high efficiency detector. 


\section{Figure Captions}

Fig. I Calculated sensitivity of the spatial neutron distribution in water to different oxygen data sets.

Fig. 2 Calculated sensitivity of the spatial neutron distribution in solid $\mathrm{CO}_{2}$ to different oxygen data sets.

Fig. 3 Calculated sensitivity of the spatial neutron distribution in liquid oxygen to different oxygen data sets.

Fig. 4 Experiment/calculation comparisons for the measured, Ref. 6, indium activations out to $65 \mathrm{~cm}$ using different oxygen data sets. All comparisons are normalized to 1.0 at $\mathrm{r}=6.5 \mathrm{~cm}$.

Fig. 5 Experiment/calculation comparisons for measured, Ref. 6, indium activations using different ${ }^{252} \mathrm{Cf}$ fission spectrum representations. All comparisons are normalized to 1.0 at $r=6.5 \mathrm{~cm}$.

Fig. 6. $\quad{ }^{252}$ Cf neutron source encapsulation used in present experiment. Fig. 7 Semi-log plot of experimental counting data. $r$ is the distance between source and detector center lines and $A$ the detector count rate $(c / s)$ normalized to the high efficiency detector. Fig. 8 Response of the zero sensitivity detector as a function of applied voltage. The operating (detector bias) of the two neutron sensitive detectors are also shown.

Fig. 9 Spatial response of the zero sensitivity detector at 875 volts bias. The ratios of the zero to low sensitivity detector count rates are shown in parentheses.

Fig: 10 Source representation in PTMG calculation: 
Fig. Il EXP/CALC comparison for oxygen deleted from the calculation. Normalization to 1.0 at $\mathrm{r}=13 \mathrm{~cm}$.

Fig. 12 EXP/CALC comparison for the modified Troubetzkoy ${ }^{7}$ evaluation for oxygen. Normalization in $13-20 \mathrm{~cm}$ region.

Fig. 13 EXP/CALC comparison for ENDF/B-I oxygen. Normalization in $13-20 \mathrm{~cm}$ region.

Fig. 14 EXP/CALC comparison for ENDF/B-III oxygen. Normalization in $13-20 \mathrm{~cm}$ region.

Fig. 15 EXP/CALC comparison for ENDF/B-IV oxygen. Normalization in 13-20 cm region.

Fig. 16 EXP/CAIC comparisons for ENDF/B-IV for two different Maxwellian neutron spectra.

Fig. 17 EXP/CALC comparison for ENDF/B-IV to best fit Maxwellian. 


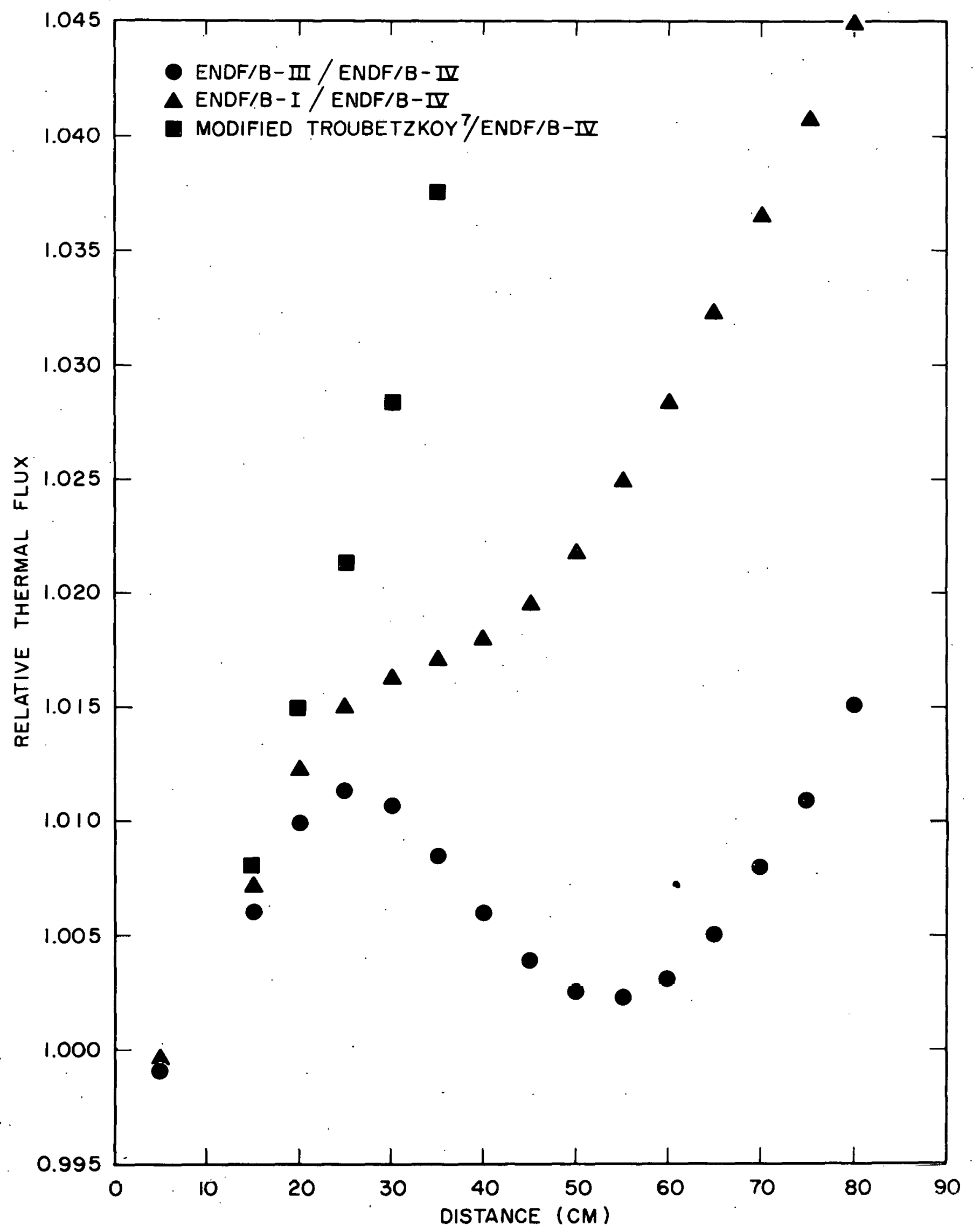

FIGURE I 


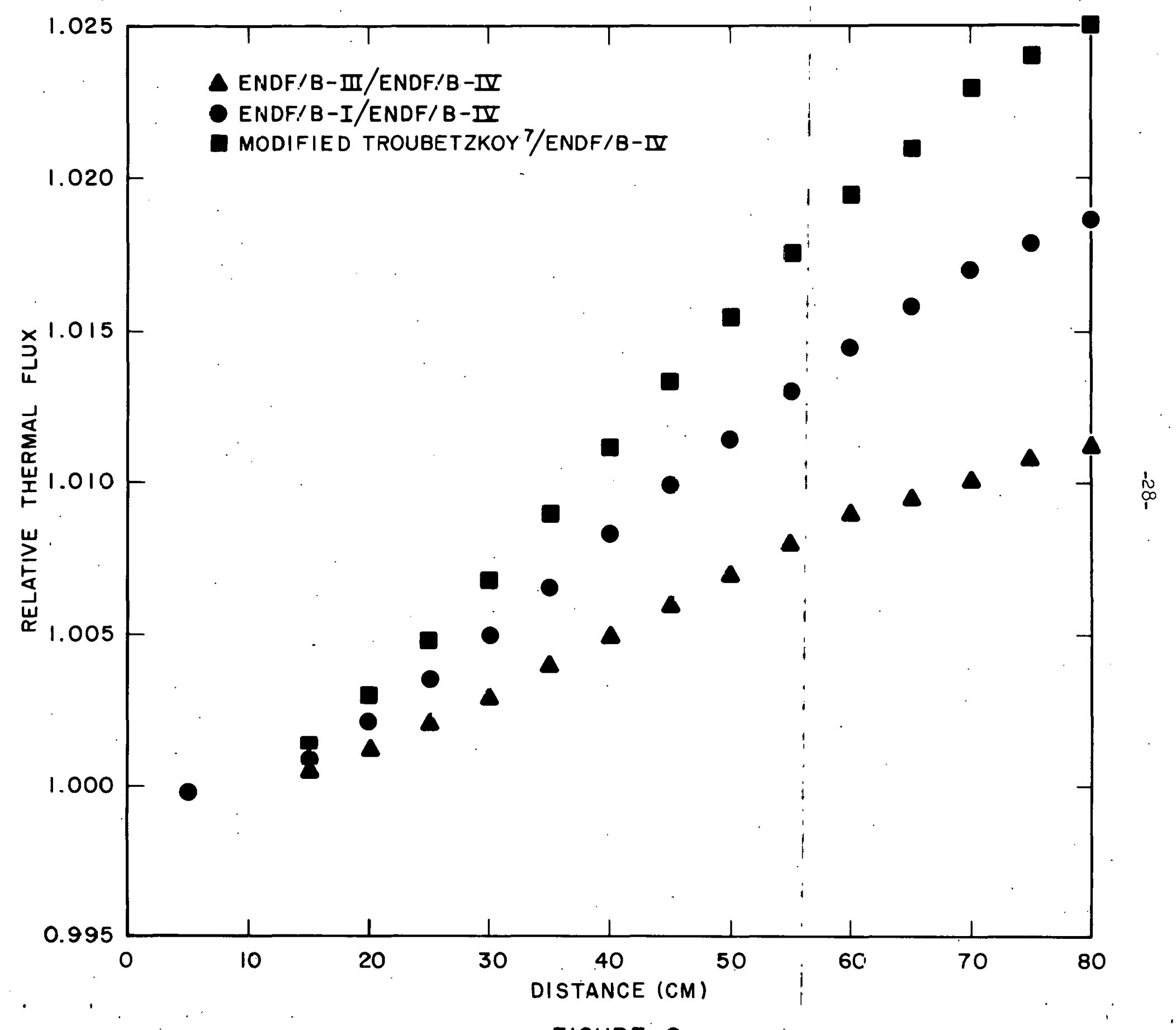




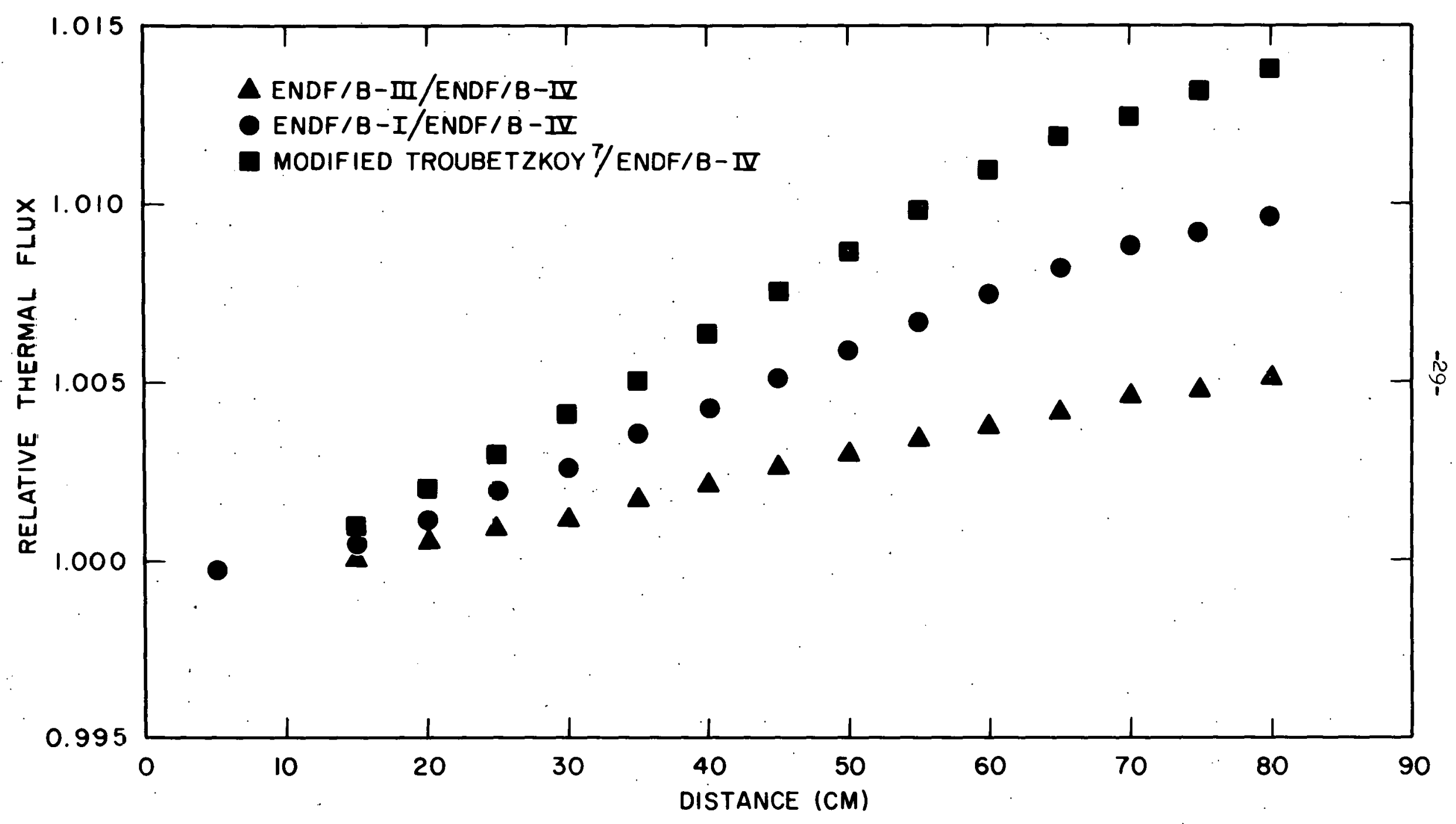

FIGURE 3 


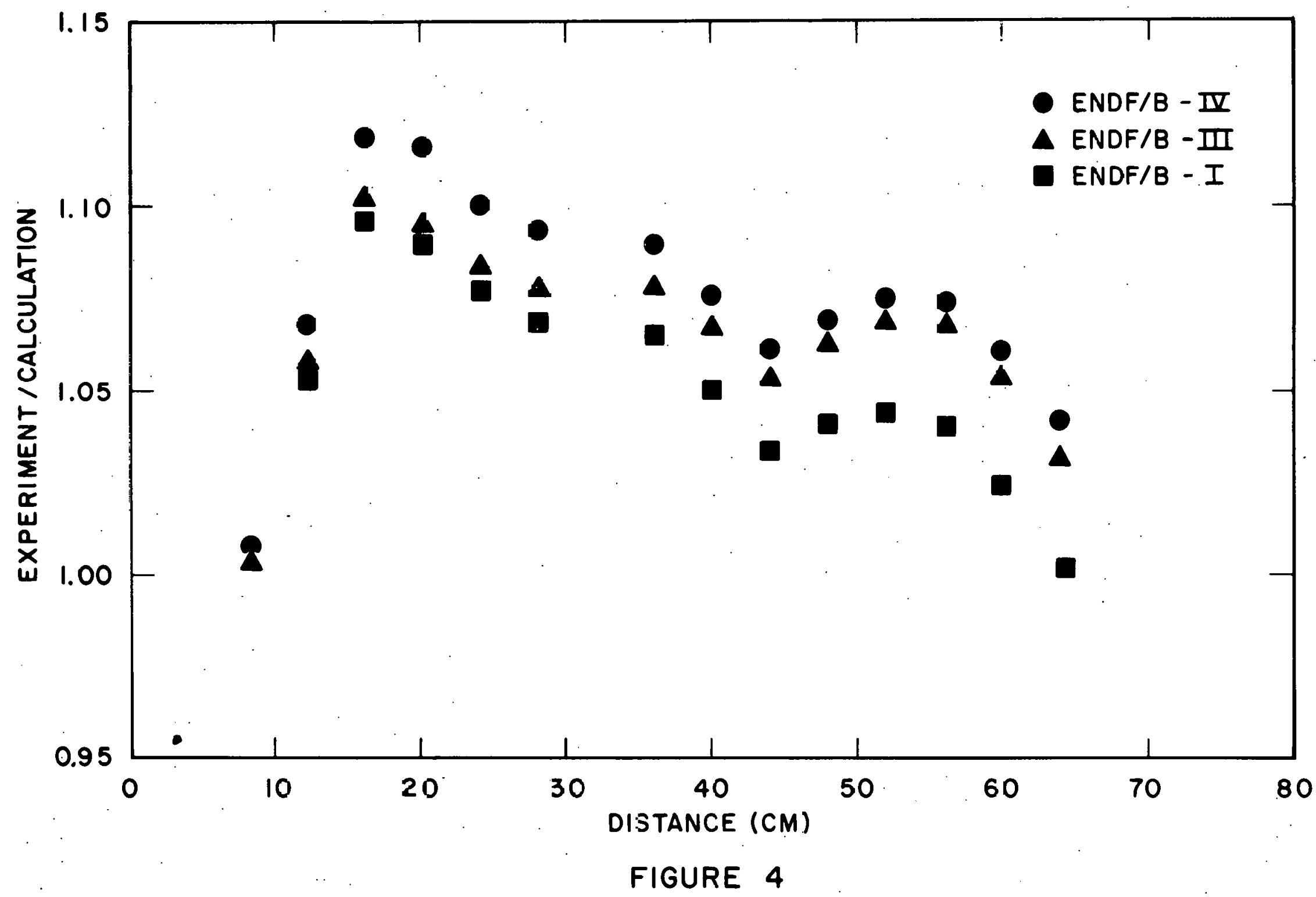




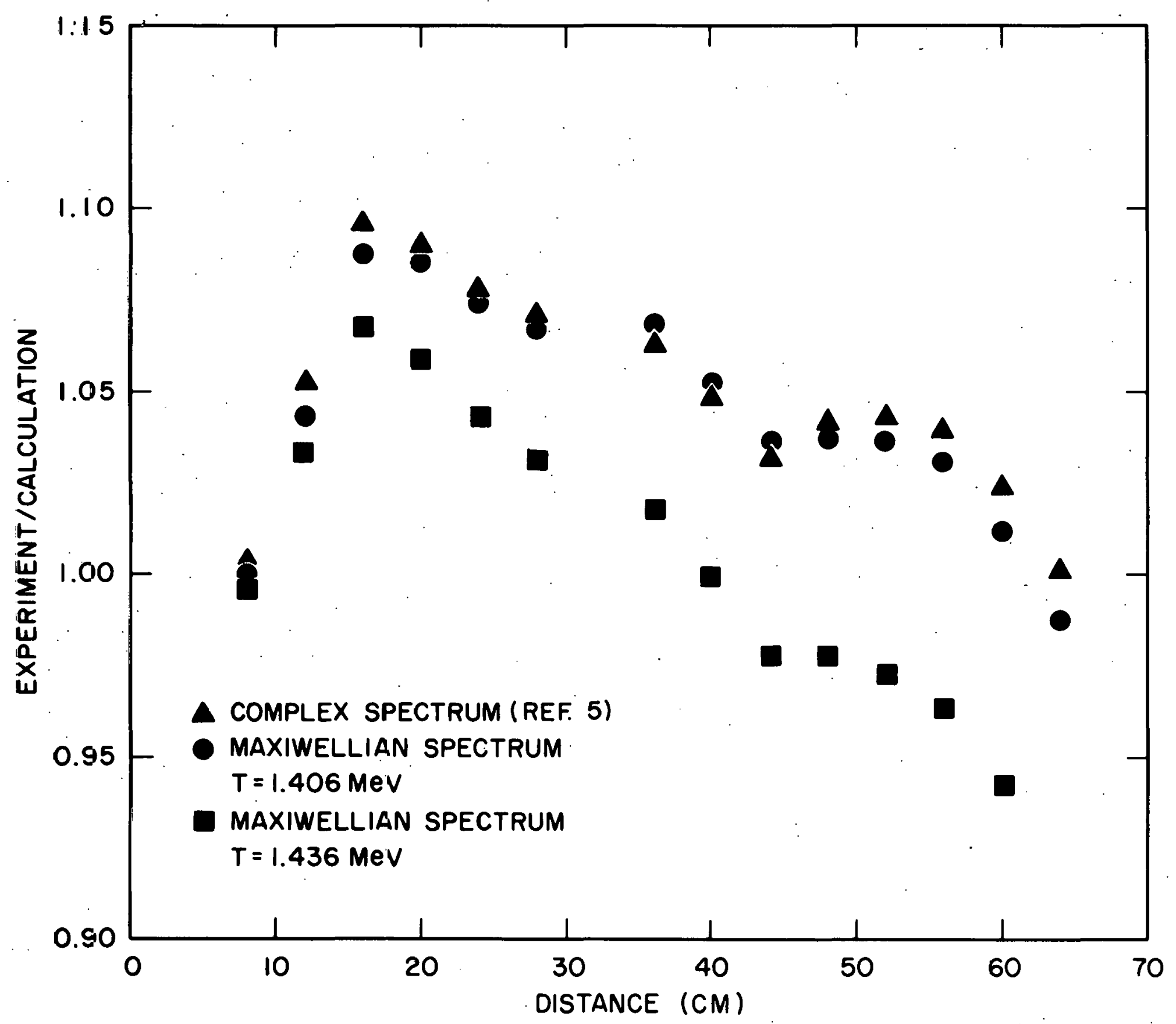

$\stackrel{\omega}{!}$

FIGURE 5 
MODEL NSD

DOUBLE ENCAPSULATED

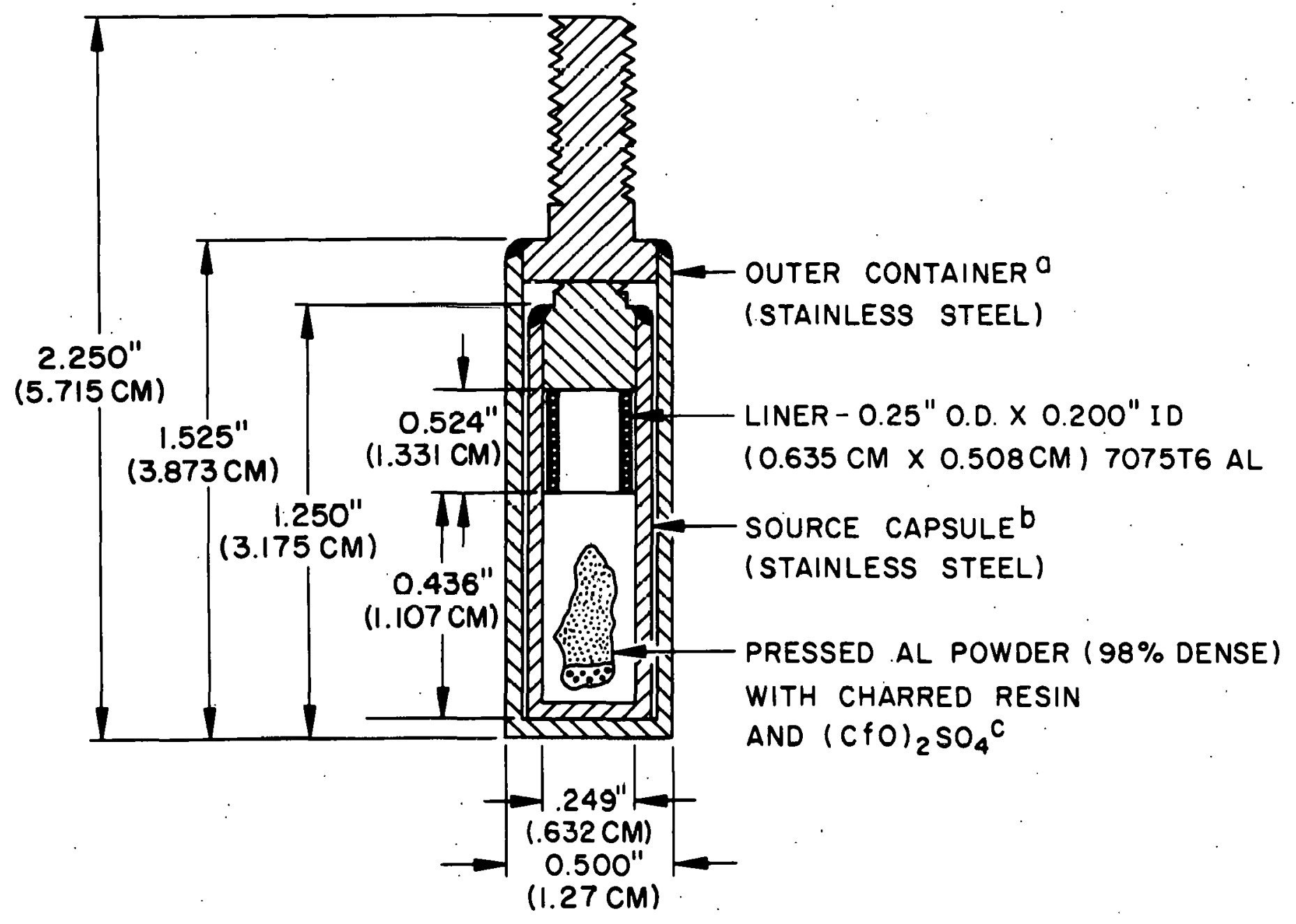

a BODY WEIGHT $15.639 \mathrm{~g}$, CAP WEIGHT $6.045 \mathrm{~g}$

b BODY WEIGHT $8.007 \mathrm{~g}$, CAP WEIGHT $4.135 \mathrm{~g}$

c $0.1 \mathrm{~g}$ DRY RESIN WITH THE FOLLOWING CALCULATED ANALYSES (w/0): C, $54.6 \% ; H, 4.6 \% ; 0,24.5 \% ; S, 16.4 \%$

FIGURE 6 
$-33-$

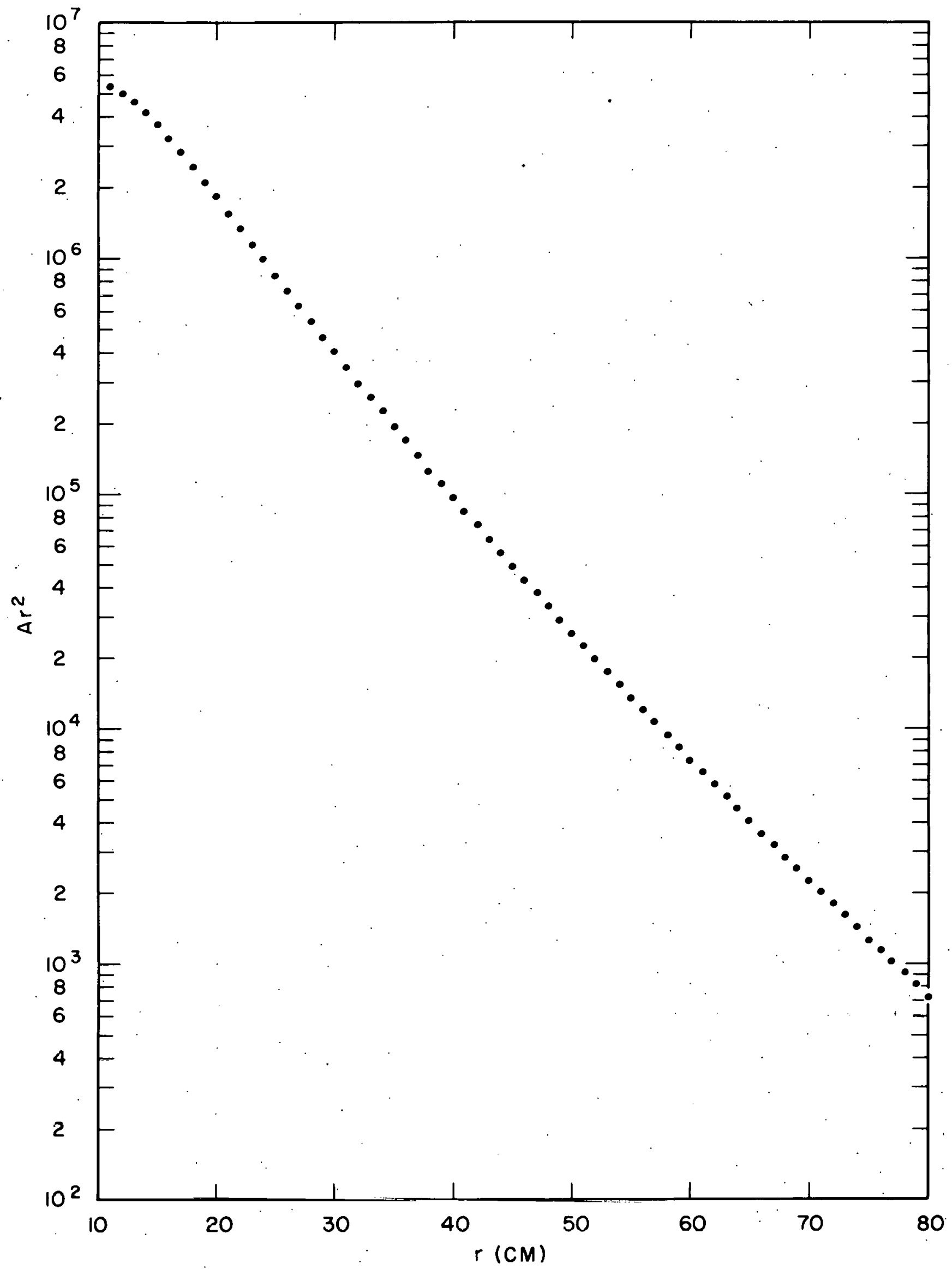

FIGURE 7 


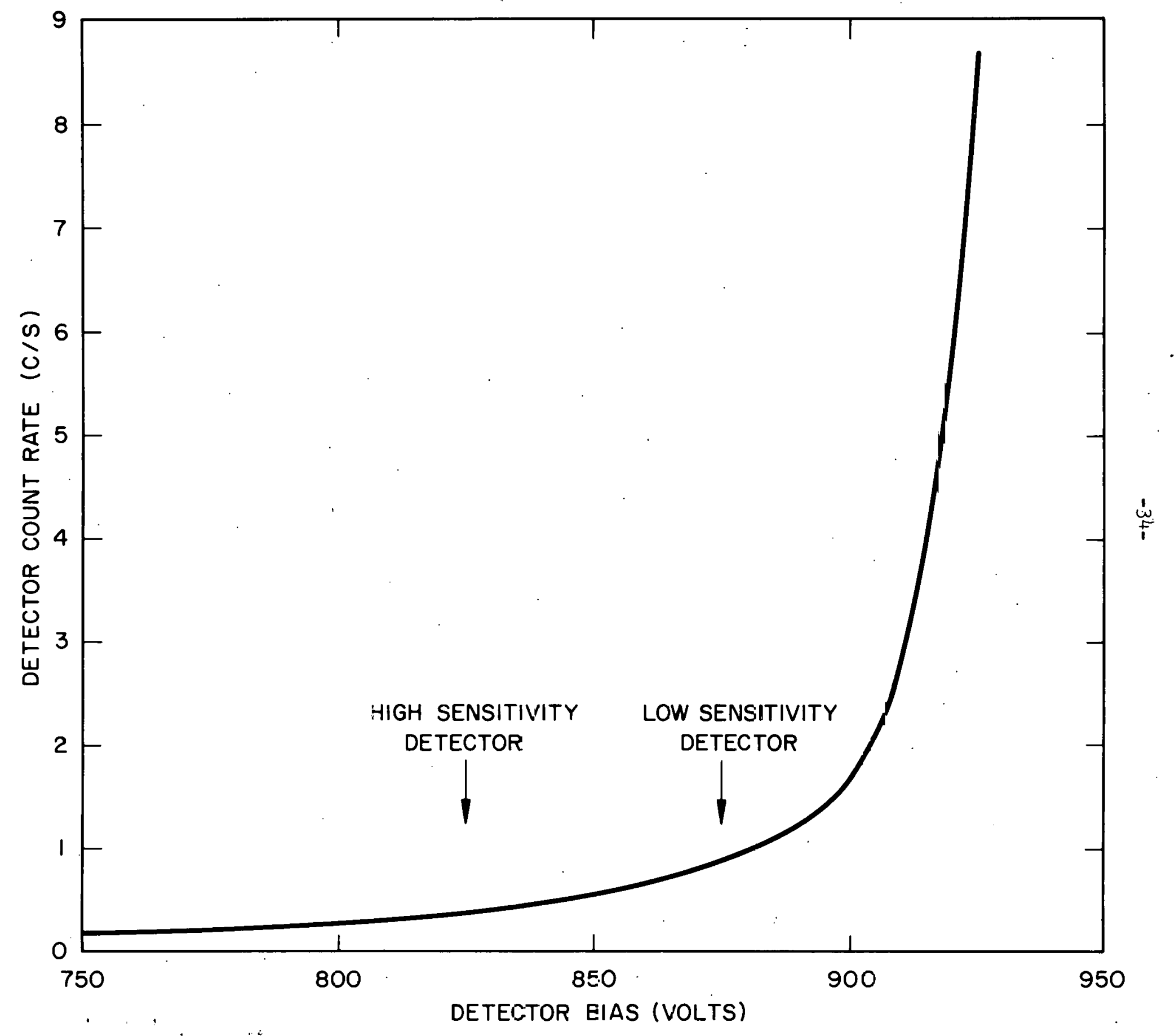




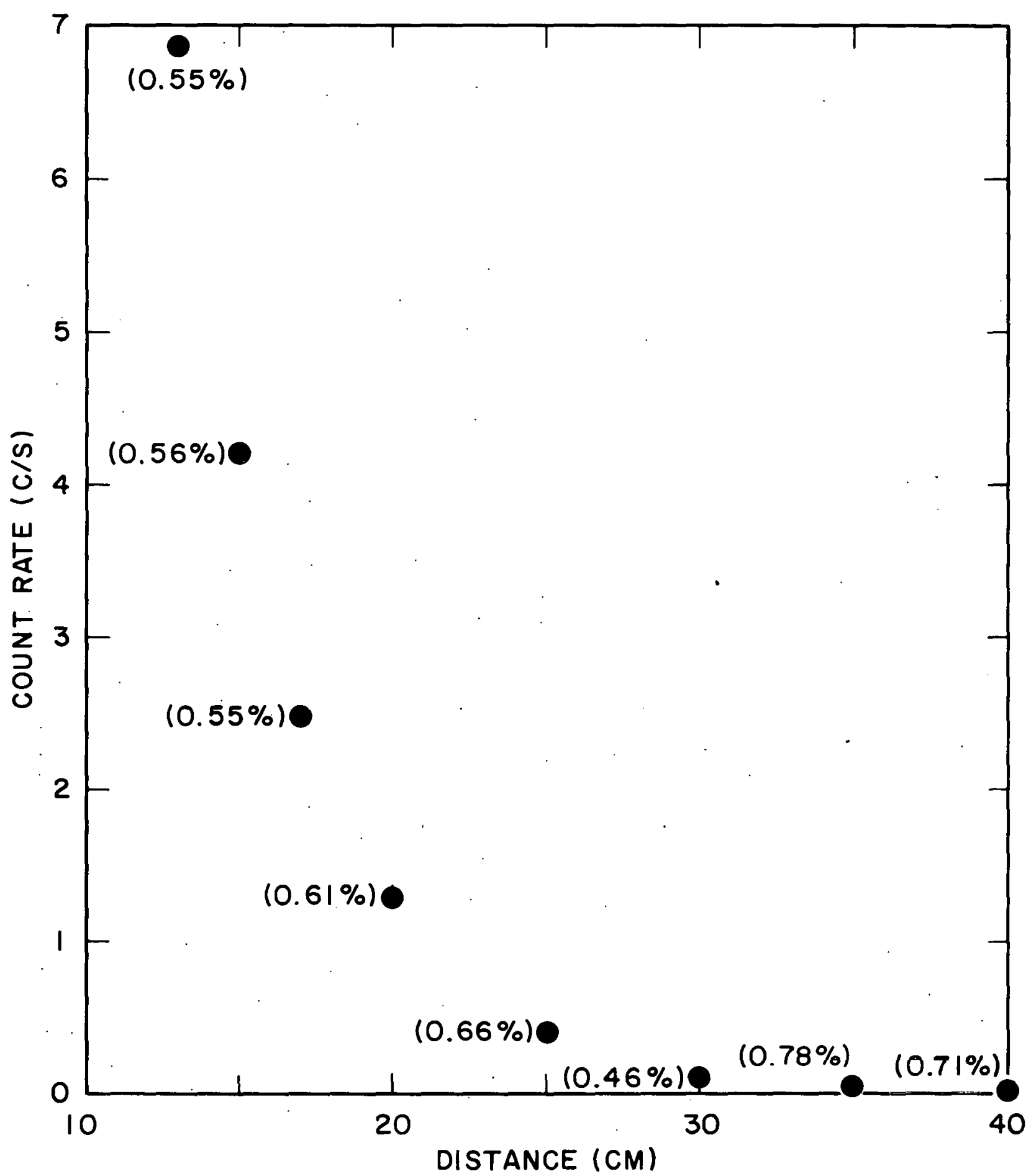

FIGURE 9 


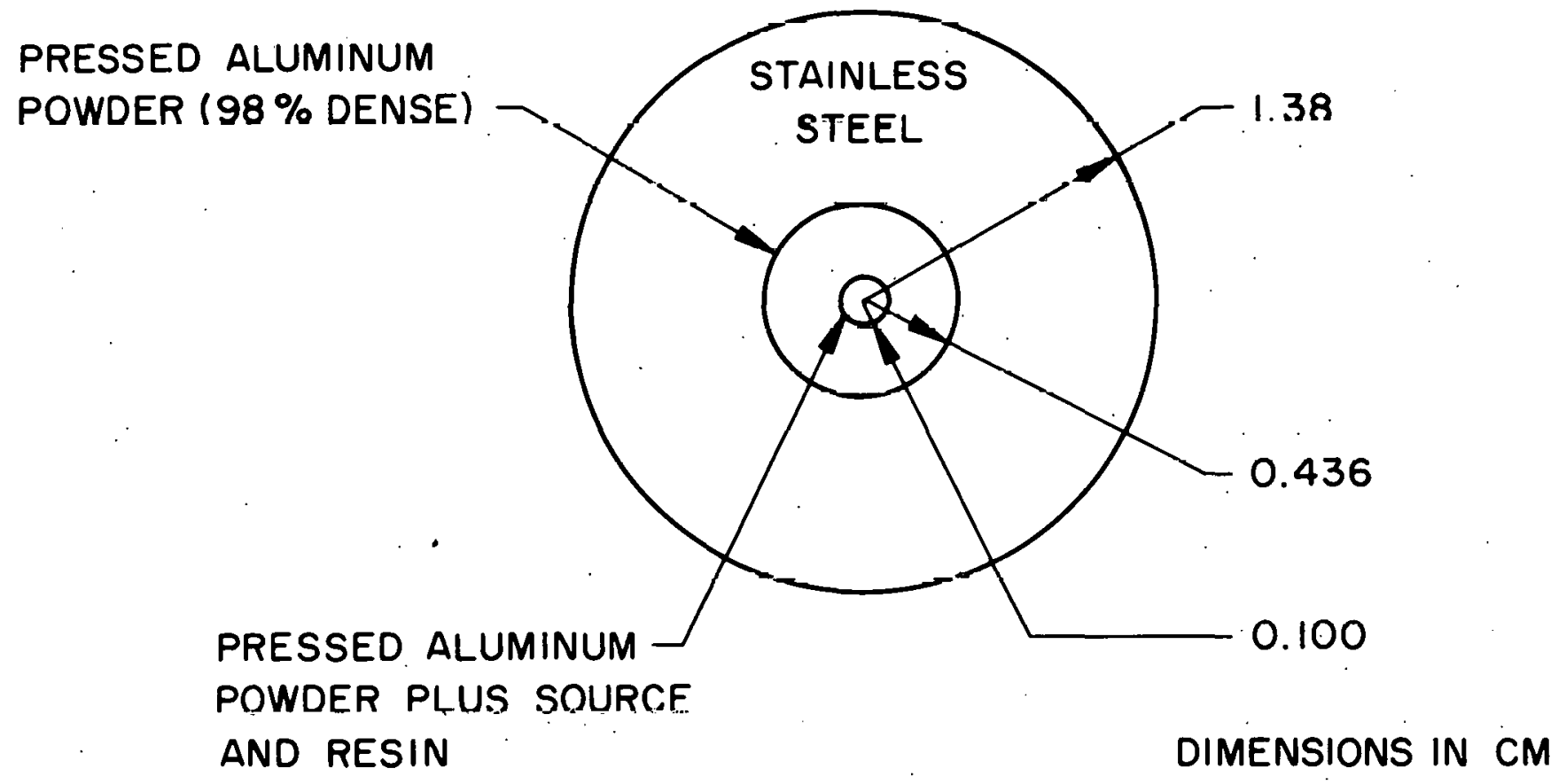

FIGURE 10 


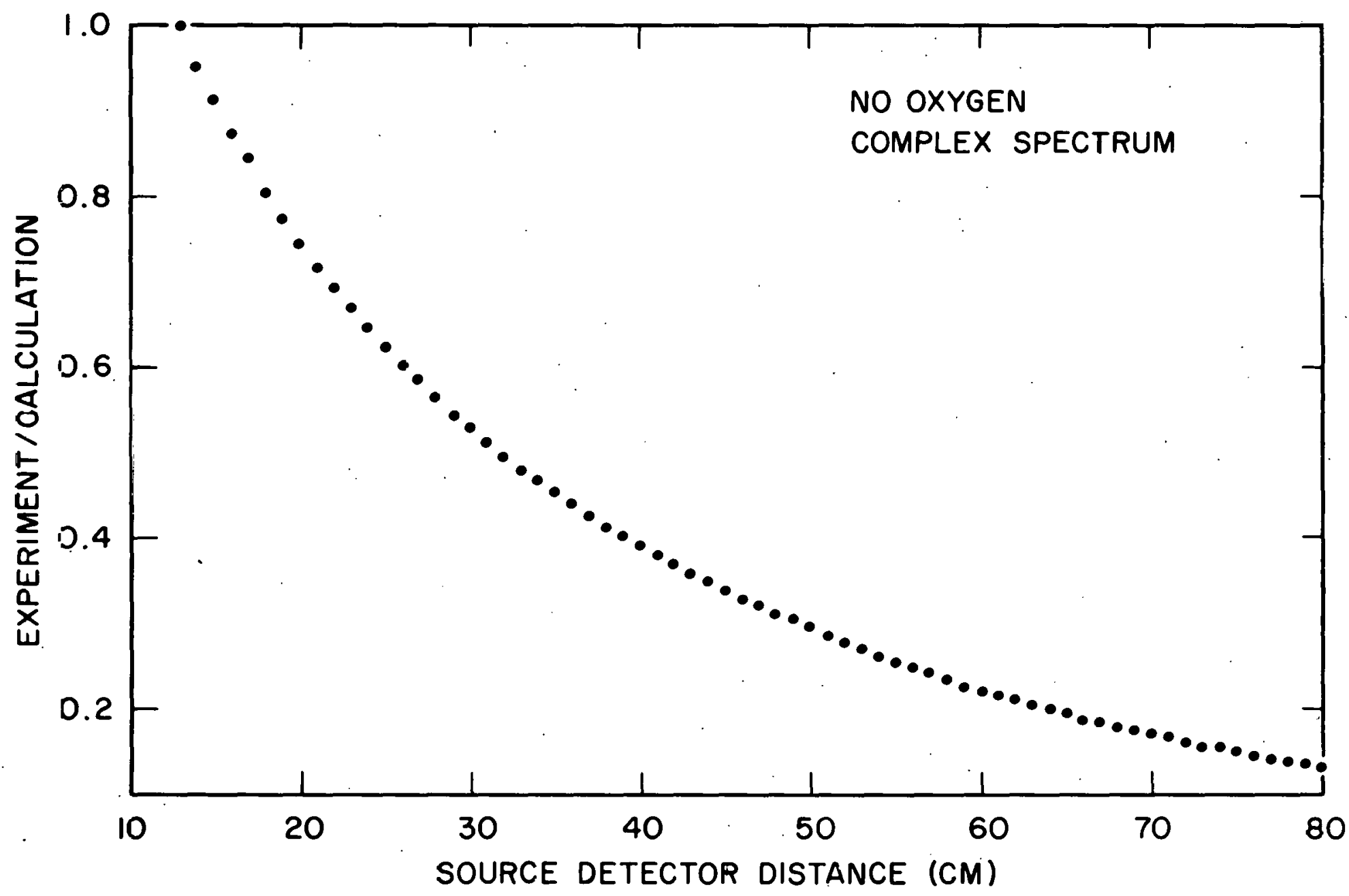

FIGURE I I 


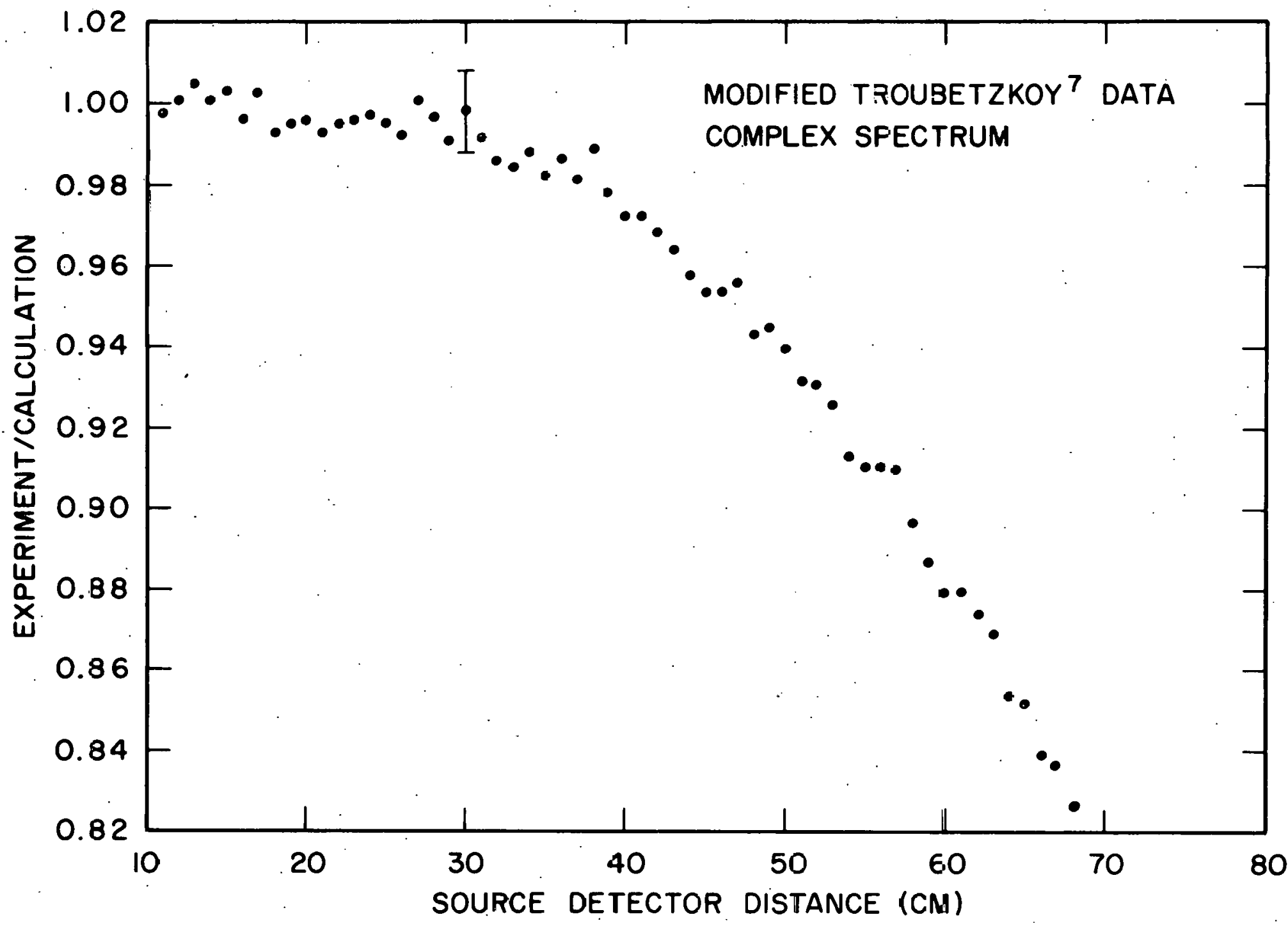

FIGURE 12 


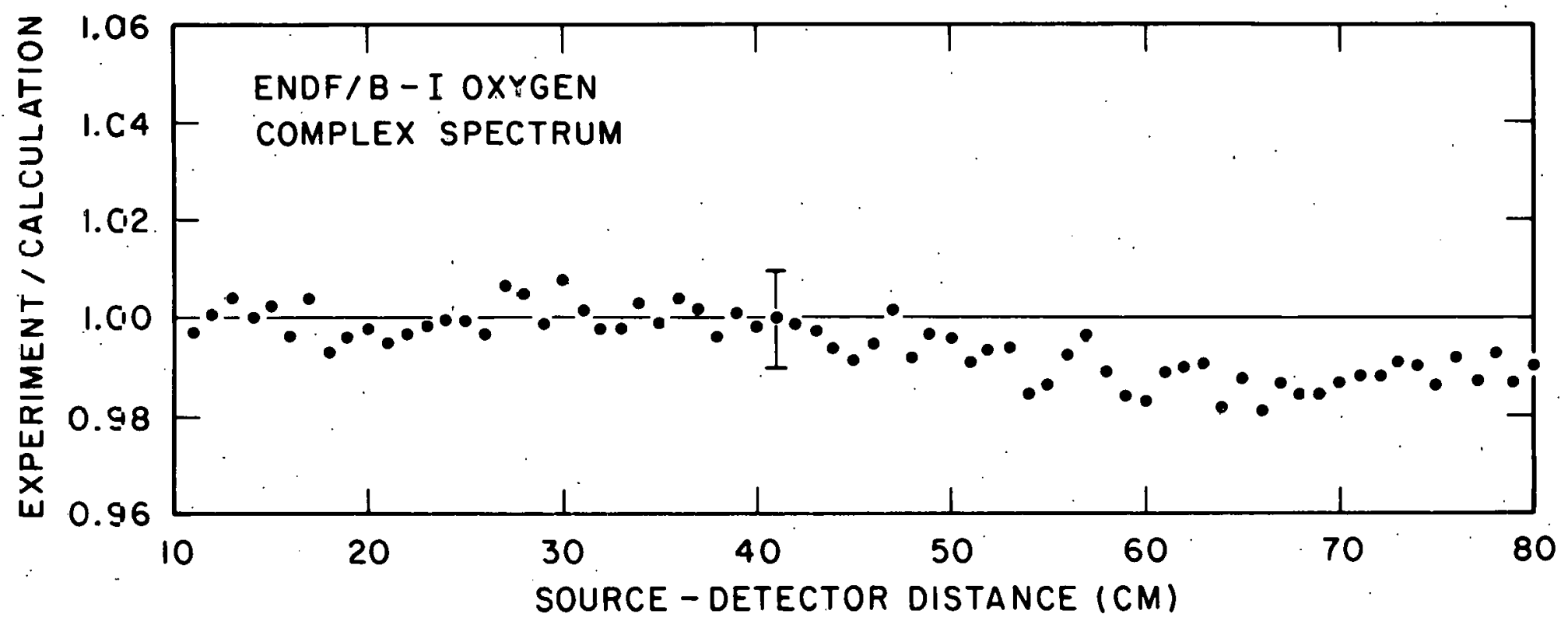

FIGURE 13 


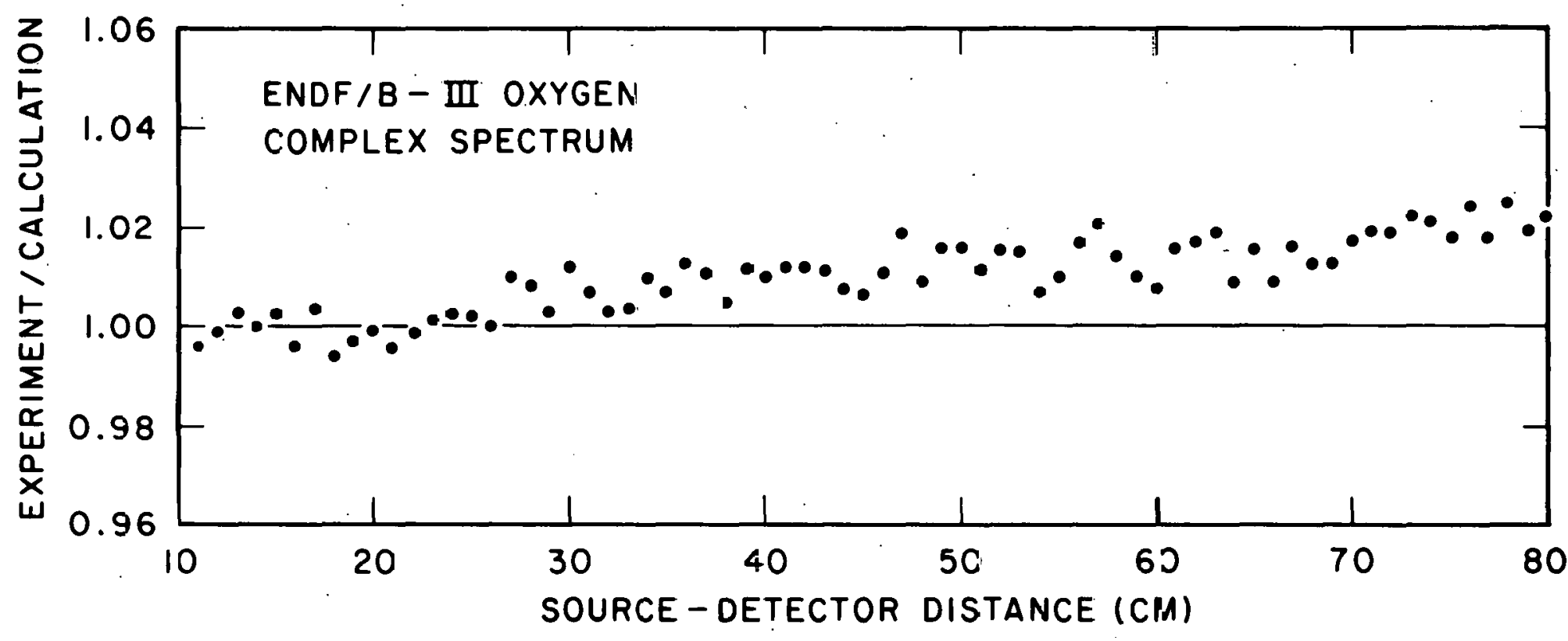

FIGURE 14 


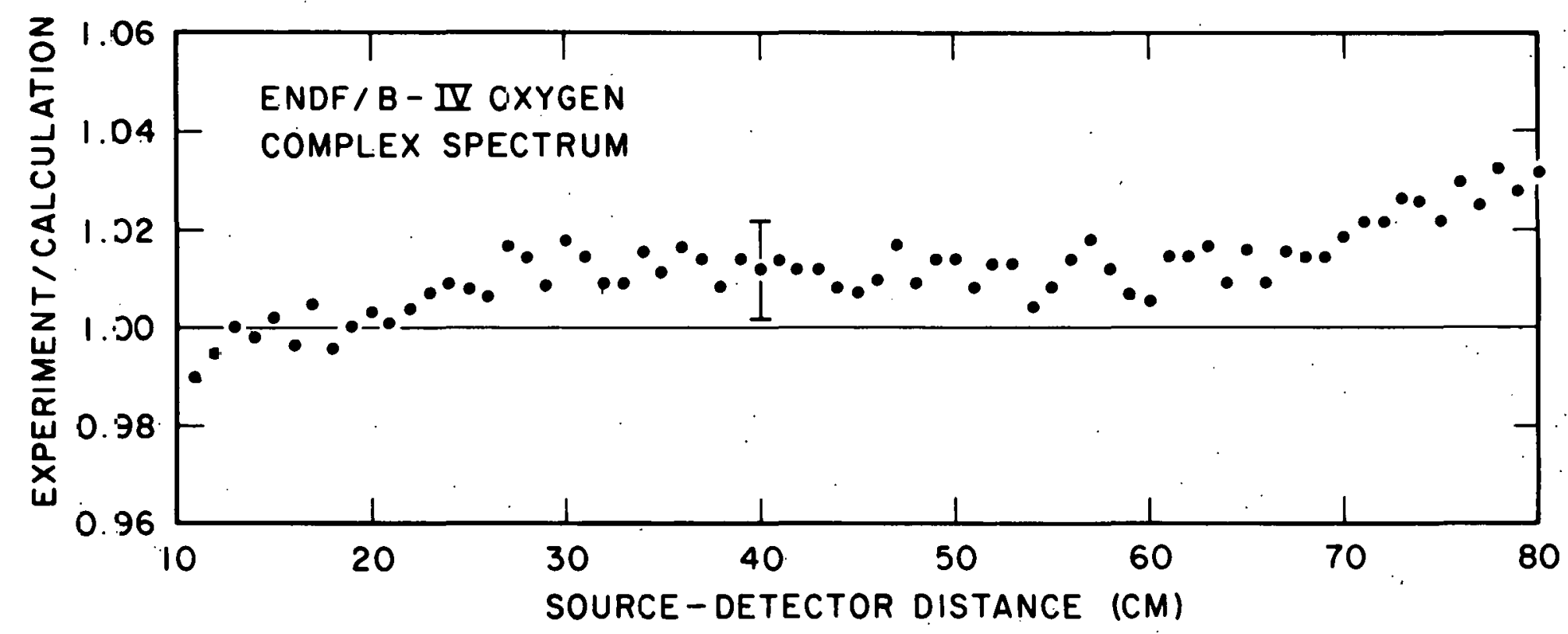

FIGURE 15 


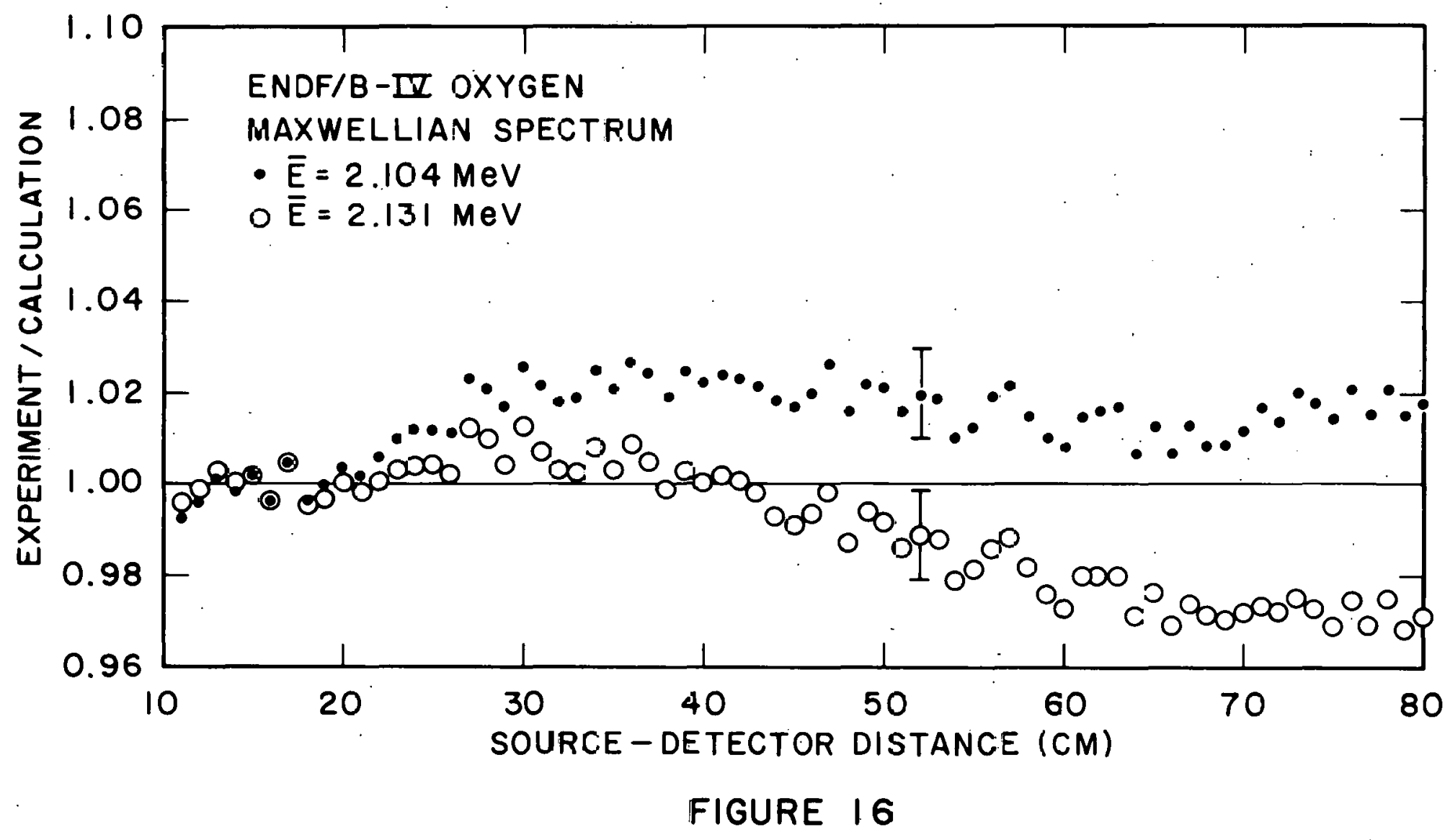




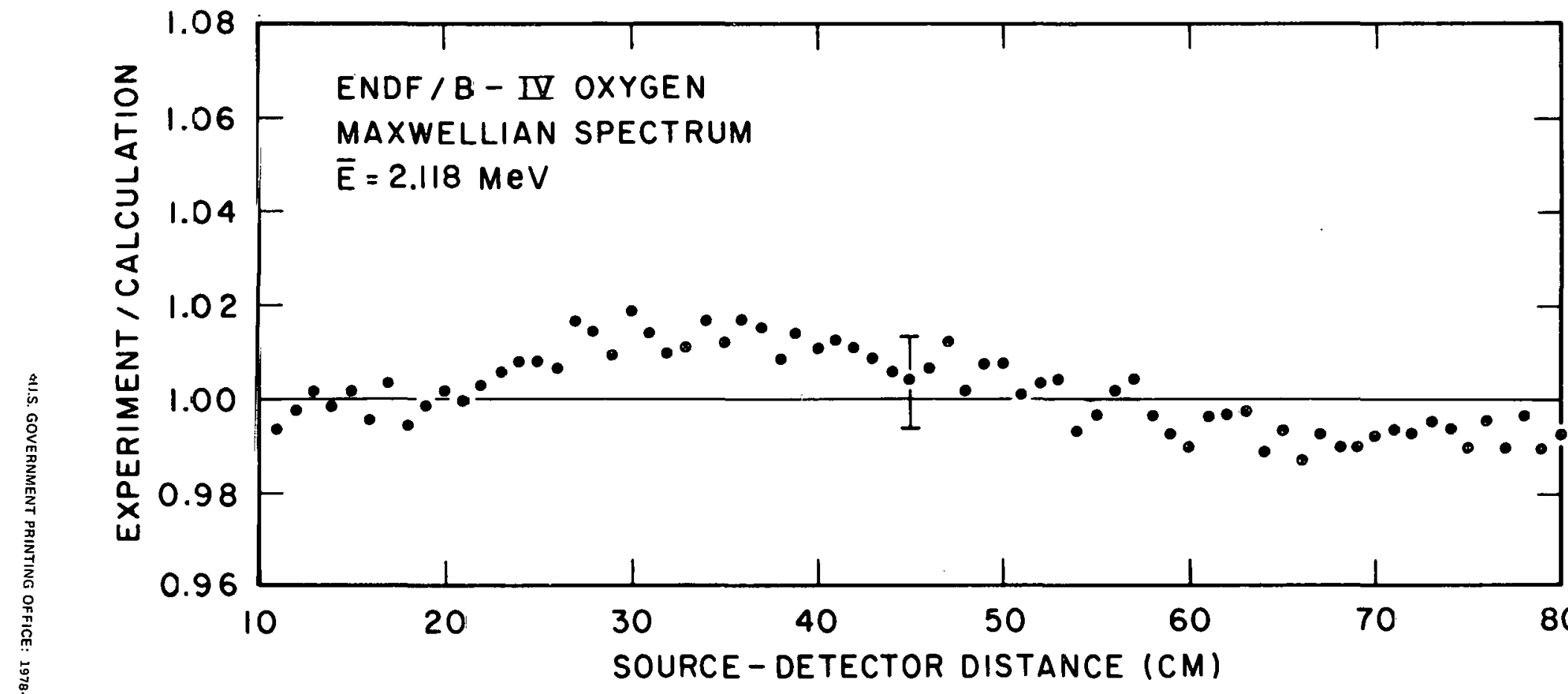

